\title{
Recurrence relations for off-shell Bethe vectors in trigonometric integrable models
}

\author{
A. Liashyk ${ }^{a, b}$ and S. Z. Pakuliak ${ }^{c, d}$ \\ a Skolkovo Institute of Science and Technology, Moscow, Russia, \\ ${ }^{b}$ NRU Higher School of Economics, Moscow, Russia, \\ E-mail: a.liashyk@gmail.com \\ ${ }^{c}$ Bogoliubov Laboratory for Theoretical Physics, JINR \\ Dubna, Moscow region, Russia \\ ${ }^{d}$ Landau School of Physics and Research, NRU MIPT \\ Dolgoprudny, Moscow region, Russia \\ E-mail: stanislav.pakuliak@jinr.ru
}

\begin{abstract}
The zero modes method is applied in order to get action of the monodromy matrix entries onto off-shell Bethe vectors in quantum integrable models associated with $U_{q}\left(\mathfrak{g l}_{N}\right)$-invariant R-matrices. The action formulas allow to get recurrence relations for off-shell Bethe vectors and for highest coefficients of the Bethe vectors scalar product.
\end{abstract}

\section{Introduction}

Algebraic Bethe ansatz [1] is a method to describe the space of states of the quantum integrable models. It is applicable, in particular, to the models defined by the monodromy matrices satisfying quadratic commutation relations and possessing special vacuum vector $|\mathrm{vac}\rangle$ in the space of states which is annihilated by the low-triangular monodromy matrix entries. A wide class of the quantum integrable models is related to the quantum groups $U_{q}(\mathfrak{g})$ [2] if structural constants of the quadratic commutation relations for the monodromy operators are $U_{q}(\mathfrak{g})$-invariant R-matrices. In this paper we consider a class of quantum trigonometric integrable models defined by the $U_{q}\left(\mathfrak{g l}_{N}\right)$-invariant R-matrices. Entries of these matrices can be written as trigonometric functions of the parameters and this is why we call such models trigonometric.

Quantum affine algebras $U_{q}(\widehat{\mathfrak{g}})$ have several descriptions which use different sets of generators. One of the description uses T-operators [3] such that their commutation relations are defined by the same $U_{q}(\mathfrak{g})$-invariant R-matrices which define monodromy matrix entries commutation relations. This coincidence opens a possibility to describe the space of states of the quantum integrable models in terms of the generators of the quantum affine algebras if one identifies the monodromy matrix of the quantum integrable model with T-operator of $U_{q}(\widehat{\mathfrak{g}})$.

In [4] a method called zero modes method was introduced for the class of supersymmetric rational $\mathfrak{g l}(m \mid n)$-invariant quantum integrable models related to the superYangian double $D Y(\mathfrak{g l}(m \mid n))$. This method uses the commutation relations between 
zero modes of the monodromy matrix entries and entries themselves in order to obtain the action of these entries onto off-shell Bethe vectors in the corresponding quantum integrable model. In the present paper we develop the zero modes method to the trigonometric quantum integrable models related to the quantum affine algebra $U_{q}\left(\widehat{\mathfrak{g l}}_{N}\right)$.

The action formulas of monodromy matrix entries onto off-shell Bethe vectors are more fundamental than explicit expressions for these vectors in terms of monodromy matrix elements. They can be used to investigate the physical quantities in the quantum integrable models such as scalar products and form-factors of the local operators without using explicit formulas for the Bethe vectors. On the other hand, the action formulas lead to the recurrent relations for the off-shell Bethe vectors, which can be solved to obtain explicit expressions for them. For the off-shell Bethe vectors in the quantum integrable models defined by $U_{q}\left(\mathfrak{g l}_{N}\right)$-invariant R-matrices two particular recurrence relations were obtained in [5] using method of the hierarchical Bethe ansatz. From viewpoint of this method these two types of the recurrence relations are related to two different ways of embedding $U_{q}\left(\mathfrak{g l}_{N-1}\right)$ monodromy matrix into $U_{q}\left(\mathfrak{g l}_{N}\right)$ monodromy: either in the upper-left corner or in the down-right corner.

To describe off-shell Bethe vectors in terms of generators of the quantum affine algebra one has to explore the Gauss decomposition of the T-operators. There are different Gauss decompositions naturally related to the embeddings mentioned above. It was shown in [6] that each of these embeddings leads to the one type recurrence relation and it is not easy combinatorial problem to prove that different type recurrence relations lead to different but equivalent presentations of the same off-shell Bethe vectors. For the rational quantum integrable models defined by the $\mathfrak{g l}(m \mid n)$-invariant R-matrices this problem was solved in [7].

Zero modes method developed in the present paper allows to find the action formulas of monodromy matrix entries onto off-shell Bethe vectors in trigonometric integrable models. This action can be used to get various recurrence relations including those obtained in [5]. Similar results were obtained in [8] for the case of the rational $\mathfrak{o}_{2 n+1^{-}}$ invariant integrable models.

The paper is composed as follows. In section 2 the quantum loop algebra $U_{q}\left(\tilde{\mathfrak{g l}}_{N}\right)$ in terms of fundamental T-operators is defined. Section 3 contains two main results of this paper, which include single and multiple actions of monodromy matrix entries onto off-shell Bethe vectors and recurrence relations for them. Section 4 is devoted to formulation of the zero modes method, which is used to obtain action formulas. Third result of the paper - the recurrence relations for the highest coefficients of the Bethe vectors scalar product is presented in the section 5. Proofs of the main propositions are gathered in two appendices.

\section{Definitions and notations}

In this paper we will explore the Cartan-Weyl generators of the quantum loop algebra $U_{q}\left(\tilde{\mathfrak{g l}}_{N}\right)$. This algebra is related to the quantum affine algebra by setting the central element in $U_{q}\left(\widehat{\mathfrak{g l}}_{N}\right)$ equal to zero. 


\section{$2.1 \quad$ R-matrix for $U_{q}\left(\tilde{\mathfrak{g l}}_{N}\right)$}

Let $N$ be dimension of the fundamental vector representation of the algebra $U_{q}\left(\mathfrak{g l}_{N}\right)$ in $\mathbb{C}^{N}$. Let $\mathrm{e}_{i j}$ be a $N \times N$ matrix unit $\left(\mathrm{e}_{i j}\right)_{k, l}=\delta_{i k} \delta_{j l}$ for $1 \leq i, j, k, l \leq N$. We introduce functions

$$
f(u, v)=\frac{q u-q^{-1} v}{u-v}, \quad g(u, v)=\frac{\left(q-q^{-1}\right) u}{u-v}, \quad \tilde{g}(u, v)=\frac{\left(q-q^{-1}\right) v}{u-v}
$$

of the arbitrary complex spectral parameters $u$ and $v$.

Define matrix $\mathbb{P}(u, v)$ acting in the tensor product $\mathbb{C}^{N} \otimes \mathbb{C}^{N}$ by the equality

$$
\mathbb{P}(u, v)=\sum_{1 \leq i, j \leq N} \mathrm{p}_{i j}(u, v) \mathrm{e}_{i j} \otimes \mathrm{e}_{j i}
$$

where rational functions $\mathrm{p}_{i j}(u, v)$ are defined as follows

$$
\mathrm{p}_{i j}(u, v)= \begin{cases}f(u, v)-1, & i=j \\ g(u, v), & i<j \\ \tilde{g}(u, v), & i>j\end{cases}
$$

Definition 2.1. Quantum trigonometric $U_{q}\left(\mathfrak{g l}_{N}\right)$-invariant $\mathrm{R}$-matrix acting in the tensor product of two fundamental vector representations of $U_{q}\left(\mathfrak{g l}_{N}\right)$ is

$$
\mathbb{R}(u, v)=\mathbb{I} \otimes \mathbb{I}+\mathbb{P}(u, v),
$$

where $\mathbb{I}=\sum_{i=1}^{N} \mathrm{e}_{i i}$ is identity matrix in $\mathbb{C}^{N}$.

\subsection{R-matrix formulation of the algebra $U_{q}\left(\tilde{\mathfrak{g l}}_{N}\right)$}

The associative algebra $U_{q}\left(\tilde{\mathfrak{g l}}_{N}\right)$ with unit $\mathbf{1}$ over $\mathbb{C}(q)$ is generated by the elements $\mathrm{T}_{i, j}^{ \pm}[ \pm m], 1 \leq i, j \leq N, m \in \mathbb{Z}_{+}$such that

$$
\mathrm{T}_{j, i}^{+}[0]=\mathrm{T}_{i, j}^{-}[0]=0, \quad i<j, \quad \mathrm{~T}_{i, i}^{+}[0] \mathrm{T}_{i, i}^{-}[0]=\mathrm{T}_{i, i}^{-}[0] \mathrm{T}_{i, i}^{+}[0]=\mathbf{1}
$$

The generators of the algebra $U_{q}\left(\tilde{\mathfrak{g l}}_{N}\right)$ may be gathered into formal series

$$
\mathrm{T}_{i, j}^{ \pm}(u)=\sum_{m=0}^{\infty} \mathrm{T}_{i, j}^{ \pm}[ \pm m] u^{\mp m}
$$

and combined in the matrices

$$
\mathrm{T}^{ \pm}(u)=\sum_{i, j=1}^{N} \mathrm{e}_{i j} \otimes \mathrm{T}_{i, j}^{ \pm}(u) \in \operatorname{End}\left(\mathbb{C}^{N}\right) \otimes U_{q}\left(\tilde{\mathfrak{g l}}_{N}\right)\left[\left[u^{\mp 1}\right]\right]
$$

which we call T-operators.

The commutation relations in the algebra $U_{q}\left(\tilde{\mathfrak{g l}}_{N}\right)$ are given by the standard RLL commutation relations

$$
\mathrm{R}(u, v) \cdot\left(\mathrm{T}^{\mu}(u) \otimes \mathbb{I}\right) \cdot\left(\mathbb{I} \otimes \mathrm{T}^{\nu}(v)\right)=\left(\mathbb{I} \otimes \mathrm{T}^{\nu}(v)\right) \cdot\left(\mathrm{T}^{\mu}(u) \otimes \mathbb{I}\right) \cdot \mathrm{R}(u, v)
$$


where $\mu, \nu= \pm$ and rational functions entering $\mathrm{R}$-matrix $\mathrm{R}(u, v)(2.3)$ should be understood as series over $v / u$ for $\mu=+, \nu=-$ and as series over $u / v$ for $\mu=-, \nu=+$. For $\mu=\nu$ these rational series can be either series over the ratio $v / u$ or the ratio $u / v$.

The commutation relations in the algebra $U_{q}(\tilde{\mathfrak{g}})$ may be written in terms of matrix entries (2.5). Using explicit expression (2.2) one gets

$$
\left[\mathrm{T}_{i, j}^{\mu}(u), \mathrm{T}_{k, l}^{\nu}(v)\right]=\mathrm{p}_{l j}(u, v) \mathrm{T}_{k, j}^{\nu}(v) \mathrm{T}_{i, l}^{\mu}(u)-\mathrm{p}_{i k}(u, v) \mathrm{T}_{k, j}^{\mu}(u) \mathrm{T}_{i, l}^{\nu}(v) .
$$

It follows from the commutation relations (2.7) or (2.8) that modes $\mathrm{T}_{i, j}^{ \pm}[m], m \geq 0$ form Borel subalgebras $U_{q}^{ \pm}\left(\tilde{\mathfrak{g l}}_{N}\right) \subset U_{q}\left(\tilde{\mathfrak{g l}}_{N}\right)$.

Remark 2.1. One can check that the restrictions to the zero mode generators (2.4) are consistent with the commutation relations (2.8).

\subsection{Gauss coordinates and the currents}

Let $\mathcal{H}$ be a representation space of the algebra $U_{q}\left(\tilde{\mathfrak{g l}}_{N}\right)$ which possesses a vector |vac $\rangle$ with following properties

$$
\mathrm{T}_{i, j}^{ \pm}(u)|\operatorname{vac}\rangle=0, \quad i>j, \quad \mathrm{~T}_{i, i}^{ \pm}(u)|\operatorname{vac}\rangle=\lambda_{i}^{ \pm}(u)|\operatorname{vac}\rangle .
$$

In what follows we will use Gauss decomposition of the T-operators of the algebra $U_{q}\left(\tilde{\mathfrak{g l}}_{N}\right)$ 9]

$$
\mathrm{T}_{i, j}^{ \pm}(u)=\sum_{\ell \leq \min (i, j)} \mathrm{F}_{j, \ell}^{ \pm}(u) k_{\ell}^{ \pm}(u) \mathrm{E}_{\ell, i}^{ \pm}(u)
$$

We call series $\mathrm{F}_{j, i}^{ \pm}(u), \mathrm{E}_{i, j}^{ \pm}(u)$ for $1 \leq i<j \leq N$ and $k_{\ell}^{ \pm}(u)$ for $1 \leq \ell \leq N$ the Gauss coordinates. Properties of the vacuum vector (2.9) are translated to

$$
\mathrm{E}_{i, j}^{ \pm}(u)|\operatorname{vac}\rangle=0, \quad i<j, \quad k_{j}^{ \pm}(u)|\operatorname{vac}\rangle=\lambda_{j}^{ \pm}(u)|\operatorname{vac}\rangle .
$$

In (2.10) we assume that $\mathrm{F}_{i, i}^{ \pm}(u)=\mathrm{E}_{i, i}^{ \pm}(u)=1$ for $1 \leq i \leq N$. Series expansion of the T-operators (2.5) imply following expansions of the Gauss coordinates

$$
\mathrm{F}_{j, i}^{ \pm}(u)=\sum_{m=0}^{\infty} \mathrm{F}_{j, i}^{ \pm}[ \pm m] u^{\mp m}, \quad \mathrm{E}_{i, j}^{ \pm}(u)=\sum_{m=0}^{\infty} \mathrm{E}_{i, j}^{ \pm}[ \pm m] u^{\mp m}, \quad k_{\ell}^{ \pm}(u)=\sum_{m=0}^{\infty} k_{\ell}^{ \pm}[ \pm m] u^{\mp m} .
$$

According to (2.4) and Gauss decomposition (2.10) $\mathrm{F}_{j, i}^{-}[0]=0$ and $\mathrm{E}_{i, j}^{+}[0]=0$.

The commutation relations between Gauss coordinates imply the commutation relations in the algebra $U_{q}\left(\tilde{\mathfrak{g l}}_{N}\right)$ in terms of the currents [9, 10]

$$
\begin{aligned}
& F_{i}(u)=\mathrm{F}_{i+1, i}^{+}(u)-\mathrm{F}_{i+1, i}^{-}(u)=\sum_{\ell \in \mathbb{Z}} \operatorname{sign}(\ell) \mathrm{F}_{i+1, i}[\ell] u^{-\ell}, \\
& E_{i}(u)=\mathrm{E}_{i, i+1}^{+}(u)-\mathrm{E}_{i, i+1}^{-}(u)=-\sum_{\ell \in \mathbb{Z}} \operatorname{sign}(-\ell) \mathrm{E}_{i, i+1}[\ell] u^{-\ell},
\end{aligned}
$$

where sign function $\operatorname{sign}(\ell)$ is defined as

$$
\operatorname{sign}(\ell)= \begin{cases}+1, & \ell \geq 0 \\ -1, & \ell<0\end{cases}
$$


The nontrivial commutation relations between currents are

$$
\begin{gathered}
k_{i}^{ \pm}(u) F_{i}(v) k_{i}^{ \pm}(u)^{-1}=\frac{q^{-1} u-q v}{u-v} F_{i}(v), \\
k_{i+1}^{ \pm}(u) F_{i}(v) k_{i+1}^{ \pm}(u)^{-1}=\frac{q u-q^{-1} v}{u-v} F_{i}(v), \\
k_{i}^{ \pm}(u)^{-1} E_{i}(v) k_{i}^{ \pm}(u)=\frac{q^{-1} u-q v}{u-v} E_{i}(v), \\
k_{i+1}^{ \pm}(u)^{-1} E_{i}(v) k_{i+1}^{ \pm}(u)=\frac{q u-q^{-1} v}{u-v} E_{i}(v), \\
\left(q^{-1} u-q v\right) F_{i}(u) F_{i}(v)=\left(q u-q^{-1} v\right) F_{i}(v) F_{i}(u), \\
\left(q u-q^{-1} v\right) E_{i}(u) E_{i}(v)=\left(q^{-1} u-q v\right) E_{i}(v) E_{i}(u), \\
(u-v) F_{i}(u) F_{i+1}(v)=\left(q^{-1} u-q v\right) F_{i+1}(v) F_{i}(u), \\
\left(q^{-1} u-q v\right) E_{i}(u) E_{i+1}(v)=(u-v) E_{i+1}(v) E_{i}(u), \\
{\left[E_{i}(u), F_{j}(v)\right]=\delta_{i, j}\left(q-q^{-1}\right) \delta(u, v)\left(k_{i+1}^{-}(v) k_{i}^{-}(v)-1\right.}
\end{gathered}
$$

and Serre relations for the currents $E_{i}(u)$ and $F_{i}(u)$ [9, 10].

In (2.15) the multiplicative delta function is defined by the formal series

$$
\delta(u, v)=\sum_{\ell \in \mathbb{Z}} \frac{u^{\ell}}{v^{\ell}}
$$

which satisfy the property

$$
\delta(u, v) G(u)=\delta(u, v) G(v)
$$

for any formal series $G(u)$.

Remark 2.2. The equalities (2.14) should be understood in a sense of equalities between formal series. It means that these commutation relations should be understood as infinite set of equalities between modes of the currents which appear after equating the coefficients at all powers $u^{\ell} v^{\ell^{\prime}}$ for $\ell, \ell^{\prime} \in \mathbb{Z}$. The rational functions in the commutation relations (2.14) should be understood as series over powers of $v / u$ in the relations containing the current $k_{j}^{+}(u)$ and over powers of $u / v$ in the relations with the current $k_{j}^{-}(u)$.

\subsection{Sets of parameters and their partitions}

It is known [11] that the Cartan-Weyl generators of $U_{q}\left(\tilde{\mathfrak{g l}}_{N}\right)$ can be identified with the generators of the currents $F_{i}(u), E_{i}(u)$ and $k_{\ell}^{ \pm}(u)$. Off-shell Bethe vectors which will be defined in the next section through these current generators [14] depend on the sets of the Bethe parameters. One needs $N-1$ types of the parameters $t_{a}^{\ell}$ for $\ell=1, \ldots, N-1$ and $a=1, \ldots, r_{\ell}$ to describe off-shell Bethe vectors for the quantum integrable model associated with $U_{q}\left(\mathfrak{g l}_{N}\right)$-invariant R-matrix. Superscript $\ell$ in $t_{a}^{\ell}$ denotes the type of the parameter while subscript $a$ counts the number of the parameters of the same type. 
We will collect parameters of the same type in the sets $\bar{t}^{\ell}=\left\{t_{1}^{\ell}, \ldots, t_{r_{\ell}}^{\ell}\right\}$ with cardinalities $\left|\bar{t}^{\ell}\right|=r_{\ell}$ and will denote collection of these sets as $\bar{t}=\left\{\bar{t}^{1}, \ldots, \bar{t}^{N-1}\right\}$. If non-negative number $r_{\ell}=0$ vanishes for some $\ell$ it means that the corresponding set $\bar{t}^{\ell}=\varnothing$ is empty. The set $\bar{t}_{a}^{\ell}$ is by definition the set $\bar{t}^{\ell} \backslash\left\{t_{a}^{\ell}\right\}$ of the cardinality $\left|\bar{t}_{a}^{\ell}\right|=r_{\ell}-1$.

Our results are formulated as sums over partitions of the sets $\bar{t}^{\ell}$ into several nonintersecting subsets. We denote these partitions as $\left\{\bar{t}_{\mathrm{I}}^{\ell}, \bar{t}_{\mathrm{II}}^{\ell}\right\} \vdash \bar{t}^{\ell}$ such that $\left|\bar{t}_{\mathrm{I}}^{\ell}\right|+\left|\bar{t}_{\mathrm{I}}^{\ell}\right|=\left|\bar{t}^{\ell}\right|$.

For any scalar functions or commuting operators of one or two variables we will use notation that $\lambda_{j}(\bar{u})$ or $f(\bar{u}, \bar{v})$ means the product of the corresponding functions over the elements of the sets $\bar{u}$ and $\bar{v}$ :

$$
\lambda_{j}^{+}(\bar{u})=\prod_{s=1}^{|\bar{u}|} \lambda_{j}^{+}\left(u_{s}\right), \quad f(\bar{u}, \bar{v})=\prod_{s=1}^{|\bar{u}|} \prod_{p=1}^{|\bar{v}|} f\left(u_{s}, v_{p}\right), \quad \mathrm{T}_{i, j}^{+}(\bar{z})=\prod_{s=1}^{|\bar{z}|} \mathrm{T}_{i, j}^{+}\left(z_{s}\right) .
$$

If any of these sets is empty then the corresponding product is equal to 1 by definition. For example, $f(\bar{u}, \varnothing) \equiv 1$.

\section{Bethe vectors and main results}

Monodromy matrix $\mathrm{T}(z)$ of any quantum integrable model with $U_{q}\left(\mathfrak{g l}_{N}\right)$-invariant Rmatrix satisfy the commutation relation (2.7) or (2.8) for $\mu=\nu$. We identify $\mathrm{T}(z) \equiv$ $\mathrm{T}^{+}(z)$. RTT commutation relations (2.7) imply that transfer matrix

$$
\mathfrak{t}(z)=\sum_{i=1}^{N} \mathrm{~T}_{i, i}(z)
$$

commutes for different values of the spectral parameters. The goal of the algebraic Bethe ansatz is to describe the solution of the eigenvalue problem

$$
\mathfrak{t}(z) \cdot \mathbb{B}(\bar{t})=\tau(z ; \bar{t}) \mathbb{B}(\bar{t})
$$

where

$$
\tau(z ; \bar{t})=\sum_{i=1}^{N} \lambda_{i}(z) f\left(z, \bar{t}^{i-1}\right) f\left(\bar{t}^{i}, z\right)
$$

and $\bar{t}$ is a set of Bethe parameters $\left\{\bar{t}^{1}, \ldots, \bar{t}^{N-1}\right\}$. The boundary sets $\bar{t}^{0}=\bar{t}^{N}=$ $\varnothing$ appearing in (3.3) are empty. The functions $\lambda_{i}(z) \equiv \lambda_{i}^{+}(z)$ are free functional parameters. In each concrete integrable model these parameters are fixed to some functions. Let $\beta_{i}(z)$ be a ratio of neighboring functional parameters

$$
\beta_{i}(z)=\frac{\lambda_{i+1}(z)}{\lambda_{i}(z)}, \quad i=1, \ldots, N-1
$$

The parameters of the Bethe vectors should satisfy Bethe equations

$$
\beta_{i}\left(t_{\ell}^{i}\right)=\frac{\lambda_{i+1}\left(t_{\ell}^{i}\right)}{\lambda_{i}\left(t_{\ell}^{i}\right)}=\frac{f\left(\bar{t}_{\ell}^{i}, t_{\ell}^{i}\right)}{f\left(t_{\ell}^{i}, \bar{t}_{\ell}^{i}\right)} \frac{f\left(t_{\ell}^{i}, \bar{t}^{i-1}\right)}{f\left(\bar{t}^{i+1}, t_{\ell}^{i}\right)}
$$


in order to fulfill eigenvalue problem (3.2). Such Bethe vectors are called on-shell.

If Bethe parameters are free then Bethe vectors are called off-shell and have the structure described by hierarchical Bethe ansatz. Off-shell Bethe vectors can be described in terms of the Cartan-Weyl generators of the quantum loop algebra $U_{q}\left(\tilde{\mathfrak{g l}}_{N}\right)$. In this case $\mathbb{B}(\bar{t}) \in \mathcal{H}$ are vectors in the representation space $\mathcal{H}$ of the algebra $U_{q}\left(\tilde{\mathfrak{g l}}_{N}\right)$.

To describe this relation one has to consider Borel subalgebras of $U_{q}^{ \pm}\left(\tilde{g l}_{N}\right) \subset U_{q}\left(\tilde{\mathfrak{g l}}_{N}\right)$ formed by the modes of T-operators $\mathrm{T}^{ \pm}(u)$ and an alternative Borel subalgebras $U_{F}$ and $U_{E}$ formed by the modes of the currents $F_{i}(u), k_{j}^{+}(u)$ and $E_{i}(u), k_{j}^{-}(u)$ respectively. One can define projections $P_{f}^{ \pm}$and $P_{e}^{ \pm}$onto intersections $U_{F}^{ \pm}=U_{F} \cap U_{q}^{ \pm}\left(\tilde{\mathfrak{g l}}_{N}\right)$ and $U_{E}^{ \pm}=U_{E} \cap U_{q}^{ \pm}\left(\tilde{\mathfrak{g l}}_{N}\right)$. These projections act on the Borel subalgebras $U_{F}$ and $U_{E}$.

Detailed investigation of these projections for quantum affine algebra $U_{q}\left(\widehat{\mathfrak{g l}}_{N}\right)$ was performed in [12]. For the purpose of the present paper we may understood projections $P_{f}^{ \pm}$acting onto product of the simple roots currents $F_{i}(t)$ as follows. To calculate projection from the product of these currents one has substitute each current by the difference of the Gauss coordinates (2.12) and then use commutation relations between them to 'normal' order all monomials such that all 'negative' Gauss coordinates $\mathrm{F}_{j, i}^{-}(t)$ are on the left of all 'positive' coordinates $\mathrm{F}_{j, i}^{+}\left(t^{\prime}\right)$. Although original expressions is a collection of monomials composed from the Gauss coordinates $\mathrm{F}_{i+1, i}^{ \pm}(t)$ only, the higher Gauss coordinates $\mathrm{F}_{j, i}^{ \pm}(t)$ for $j>i+1$ will appear due to this normal ordering process. Then application of the projection $P_{f}^{+}$means removing of all monomials which have at least one 'negative' coordinate on the left. Analogously, application of the projection $P_{f}^{-}$means removing of all monomials which have at least one 'positive' coordinate on the right. Projections $P_{e}^{ \pm}$acting onto product of the currents $E_{i}(t)$ can be understood similarly according to the cyclic ordering of the Cartan-Weyl generators (see details in [12, 13]). In [14 more effective methods to calculate such projections were developed. We address an interested reader to this paper and reference therein.

Let us introduce the ordered products of the simple root currents $\mathcal{F}_{i}\left(\bar{t}^{i}\right)$

$$
\mathcal{F}_{i}\left(\bar{t}^{i}\right)=\prod_{\ell<\ell^{\prime}}^{r_{i}} f\left(t_{\ell^{\prime}}^{i}, t_{\ell}^{i}\right) F_{i}\left(t_{1}^{i}\right) F_{i}\left(t_{1}^{i}\right) \cdots F_{i}\left(t_{r_{i}}^{i}\right) .
$$

Each $\mathcal{F}_{i}\left(\bar{t}^{i}\right)$ is obviously symmetric with respect to permutations of the elements in the set $\bar{t}^{i}$ due to the commutation relations (2.14). In order to express off-shell Bethe vectors in terms of the Cartan-Weyl generators of the algebra $U_{q}\left(\tilde{g l}_{N}\right)$ we define the normalized ordered product

$$
\mathbb{F}(\bar{t})=\prod_{i=1}^{N-2} f\left(\bar{t}^{i+1}, \bar{t}^{i}\right)^{-1} \mathcal{F}_{1}\left(\bar{t}^{1}\right) \mathcal{F}_{2}\left(\bar{t}^{2}\right) \cdots \mathcal{F}_{N-1}\left(\bar{t}^{N-1}\right)
$$

We call projection of this product of currents $P_{f}^{+}(\mathbb{F}(\bar{t}))$ off-shell pre-Bethe vector and off-shell Bethe vector $\mathbb{B}(\bar{t})$ itself is [14]

$$
\mathbb{B}(\bar{t})=\mathbb{B}\left(\bar{t}^{1}, \bar{t}^{2}, \ldots, \bar{t}^{N-1}\right)=P_{f}^{+}(\mathbb{F}(\bar{t}))|\mathrm{vac}\rangle .
$$

The commutation relations of the currents and properties of the projections imply that if any of the set of the Bethe parameters $\bar{t}^{i}=\varnothing$ is empty then pre-Bethe vector 
factorizes into product of pre-Bethe vectors for $U_{q}\left(\tilde{\mathfrak{g l}}_{i}\right)$ and $U_{q}\left(\tilde{\mathfrak{g l}}_{N-i}\right)$ algebras

$$
P_{f}^{+}\left(\mathbb{F}\left(\bar{t}^{1}, \ldots, \bar{t}^{i-1}, \varnothing, \bar{t}^{i+1}, \ldots, \bar{t}^{N-1}\right)\right)=P_{f}^{+}\left(\mathbb{F}\left(\bar{t}^{1}, \ldots, \bar{t}^{i-1}\right)\right) \cdot P_{f}^{+}\left(\mathbb{F}\left(\bar{t}^{i+1}, \ldots, \bar{t}^{N-1}\right)\right) .
$$

In particular, when $i=1$ or $i=N-1$ the off-shell Bethe vectors $\mathbb{B}\left(\varnothing, \bar{t}^{2}, \ldots, \bar{t}^{N-1}\right)$ and $\mathbb{B}\left(\bar{t}^{1}, \ldots, \bar{t}^{N-2}, \varnothing\right)$ are $U_{q}\left(\tilde{\mathfrak{g l}}_{N-1}\right)$ Bethe vectors.

\subsection{Action formulas}

In addition to the rational functions (2.1) we introduce the functions

$$
h(u, v)=\frac{f(u, v)}{g(u, v)}, \quad \tilde{h}(u, v)=\frac{f(u, v)}{\tilde{g}(u, v)}
$$

which satisfy the properties

$$
1-\frac{q^{-1}}{f(u, v)}=\frac{1}{h(u, v)} \quad \text { or } \quad h(u, v)-\frac{q^{-1}}{g(u, v)}=1
$$

and

$$
1-\frac{q}{f(u, v)}=\frac{1}{\tilde{h}(u, v)} \quad \text { or } \quad \tilde{h}(u, v)-\frac{q}{\tilde{g}(u, v)}=1 .
$$

For two sets $\bar{x}$ and $\bar{y}$ of the same cardinalities $|\bar{x}|=|\bar{y}|=n$ one can define the Izergin determinant

$$
\mathrm{K}(\bar{x} \mid \bar{y})=\frac{\prod_{i=1}^{n} x_{i} \prod_{1 \leq i, j \leq n}\left(q x_{i}-q^{-1} y_{j}\right)}{\prod_{1 \leq i<j \leq n}\left(x_{i}-x_{j}\right)\left(y_{j}-y_{i}\right)} \operatorname{det}\left[\frac{q-q^{-1}}{\left(x_{i}-y_{j}\right)\left(q x_{i}-q^{-1} y_{j}\right)}\right]
$$

and

$$
\tilde{\mathrm{K}}(\bar{x} \mid \bar{y})=\prod_{i=1}^{n} \frac{y_{i}}{x_{i}} \mathrm{~K}(\bar{x} \mid \bar{y})
$$

Note that for $n=1$

$$
\mathrm{K}(x \mid y)=g(x, y) \quad \text { and } \quad \tilde{\mathrm{K}}(x \mid y)=\tilde{g}(x, y) .
$$

First main result of the paper can be formulated as following

Proposition 3.1. Let $\bar{z}=\left\{z_{1}, \ldots, z_{r}\right\}$ be a set of arbitrary parameters of cardinality $|\bar{z}|=r$. Then, the multiple action of monodromy matrix elements $\mathrm{T}_{i, j}(\bar{z})$ onto off-shell Bethe vector $\mathbb{B}(\bar{t})$ is given by the formula

$$
\begin{aligned}
\mathrm{T}_{i, j}(\bar{z}) \mathbb{B}(\bar{t}) & =\lambda_{1}(\bar{z}) \sum_{\text {part }} \mathbb{B}\left(\bar{w}_{\mathrm{II}}\right) \frac{\prod_{p=j}^{i-1} f\left(\bar{w}_{\mathrm{I}}^{p}, \bar{w}_{\mathrm{II}}^{p}\right)}{\prod_{p=j}^{i-2} f\left(\bar{w}_{\mathrm{I}}^{p+1}, \bar{w}_{\mathrm{III}}^{p}\right)} \times \\
& \times \prod_{p=1}^{i-1} \frac{\beta_{p}\left(w_{\mathrm{I}}^{p}\right) \mathrm{K}\left(\bar{w}_{\mathrm{I}}^{p} \mid \bar{w}_{\mathrm{I}}^{p-1}\right) f\left(\bar{w}_{\mathrm{I}}^{p}, \bar{w}_{\mathrm{II}}^{p}\right)}{f\left(\bar{w}_{\mathrm{I}}^{p}, \bar{w}_{\mathrm{I}}^{p-1}\right) f\left(\bar{w}_{\mathrm{I}}^{p}, \bar{w}_{\mathrm{II}}^{p-1}\right)} \frac{\tilde{\mathrm{K}}\left(\bar{w}_{\mathrm{II}}^{p+1} \mid \bar{w}_{\mathrm{III}}^{p}\right) f\left(\bar{w}_{\mathrm{II}}^{p}, \bar{w}_{\mathrm{III}}^{p}\right)}{f\left(\bar{w}_{\mathrm{II}}^{p+1}, \bar{w}_{\mathrm{III}}^{p}\right) f\left(\bar{w}_{\mathrm{II}}^{p+1}, \bar{w}_{\mathrm{II}}^{p}\right)}
\end{aligned}
$$

where sum in (3.15) goes over partitions described below: 
- $\bar{w}^{p}=\left\{\bar{z}, \bar{t}^{p}\right\}$. These sets will be divided in subsets $\left\{\bar{w}_{\mathrm{I}}^{p}, \bar{w}_{\mathrm{II}}^{p}, \bar{w}_{\mathrm{III}}^{p}\right\} \vdash \bar{w}^{p}$.

- Boundary conditions: $\bar{w}_{\mathrm{I}}^{0}=\bar{w}_{\mathrm{II}}^{N}=\{\bar{z}\}, \bar{w}_{\mathrm{II}}^{0}=\bar{w}_{\mathrm{II}}^{0}=\bar{w}_{\mathrm{I}}^{N}=\bar{w}_{\mathrm{II}}^{N}=\varnothing$.

- Subsets $\bar{w}_{\mathrm{I}}^{p}$ are non empty only for $p<i$ and have cardinality $\left|\bar{w}_{\mathrm{I}}^{s}\right|=r$.

- Subsets $\bar{w}_{\mathrm{III}}^{p}$ are non empty only for $p \geq j$ and have cardinality $\left|\bar{w}_{\mathrm{II}}^{s}\right|=r$.

This proposition is a direct consequence of the single action formulated in the following

Proposition 3.2. The single monodromy matrix entry $\mathrm{T}_{i, j}(z)$ action onto off-shell Bethe vector is given by the exporession

$$
\mathrm{T}_{i, j}(z) \mathbb{B}(\bar{t})=\lambda_{1}(z) \sum_{\text {part }} \mathbb{B}\left(\bar{w}_{\mathrm{II}}\right) \mathcal{A}_{i, j}\left(\bar{w}_{\mathrm{I}} ; \bar{w}_{\mathrm{II}} ; \bar{w}_{\mathrm{III}}\right),
$$

where

$$
\begin{aligned}
\mathcal{A}_{i, j}\left(\bar{w}_{\mathrm{I}} ; \bar{w}_{\mathrm{II}} ; \bar{w}_{\mathrm{III}}\right) & =\frac{\prod_{p=j}^{i-1} f\left(\bar{w}_{\mathrm{I}}^{p}, \bar{w}_{\mathrm{II}}^{p}\right)}{\prod_{p=j}^{i-2} f\left(\bar{w}_{\mathrm{I}}^{p+1}, \bar{w}_{\mathrm{II}}^{p}\right)} \times \\
& \times \prod_{p=1}^{i-1} \frac{\beta_{p}\left(\bar{w}_{\mathrm{I}}^{p}\right) f\left(\bar{w}_{\mathrm{I}}^{p}, \bar{w}_{\mathrm{II}}^{p}\right)}{h\left(\bar{w}_{\mathrm{I}}^{p}, \bar{w}_{\mathrm{I}}^{p-1}\right) f\left(\bar{w}_{\mathrm{I}}^{p}, \bar{w}_{\mathrm{II}}^{p-1}\right)} \prod_{p=j}^{N-1} \frac{f\left(\bar{w}_{\mathrm{II}}^{p}, \bar{w}_{\mathrm{II}}^{p}\right)}{\tilde{h}\left(\bar{w}_{\mathrm{II}}^{p+1}, \bar{w}_{\mathrm{II}}^{p}\right) f\left(\bar{w}_{\mathrm{II}}^{p+1}, \bar{w}_{\mathrm{III}}^{p}\right)}
\end{aligned}
$$

and sum in (3.15) goes over partitions described in proposition 3.1 for $r=1$.

Proofs of the propositions 3.2 can be found in the appendices $\mathrm{A}$. Proof of proposition 3.1 is similar to the proof of analogous statement given in appendix B of the paper [4] and based on the properties of the Izergin determinant (3.12).

Corollary 3.3. For $i \leq j$ the action of the matrix entries $\mathrm{T}_{i, j}(z)$ onto off-shell Bethe vectors is simplified to

$$
\mathrm{T}_{i, j}(z) \mathbb{B}(\bar{t})=\lambda_{1}(z) \sum_{\text {part }} \mathbb{B}\left(\bar{w}_{\mathrm{II}}\right) \prod_{p=1}^{i-1} \frac{\beta_{p}\left(\bar{w}_{\mathrm{I}}^{p}\right) f\left(\bar{w}_{\mathrm{I}}^{p}, \bar{w}_{\mathrm{II}}^{p}\right)}{h\left(\bar{w}_{\mathrm{I}}^{p}, \bar{w}_{\mathrm{I}}^{p-1}\right) f\left(\bar{w}_{\mathrm{I}}^{p}, \bar{w}_{\mathrm{II}}^{p-1}\right)} \prod_{p=j}^{N-1} \frac{f\left(\bar{w}_{\mathrm{II}}^{p}, \bar{w}_{\mathrm{II}}^{p}\right)}{\tilde{h}\left(\bar{w}_{\mathrm{II}}^{p+1}, \bar{w}_{\mathrm{II}}^{p}\right) f\left(\bar{w}_{\mathrm{II}}^{p+1}, \bar{w}_{\mathrm{III}}^{p}\right)} .
$$

\subsection{Recurrence relations}

To formulate the second main result of this paper we introduce the notation $\left\{\bar{t}^{s}\right\}_{i}^{j}$ which means collection of the sets $\left\{\bar{t}^{i}, \bar{t}^{i+1}, \ldots, \bar{t}^{j}\right\}$.

Proposition 3.4. Off-shell Bethe vectors $\mathbb{B}(\bar{t})$ satisfy the recurrent relations

$$
\begin{aligned}
\mathbb{B}\left(\left\{\bar{t}^{s}\right\}_{1}^{\ell-1},\left\{z, \bar{t}^{\ell}\right\},\left\{\bar{t}^{s}\right\}_{\ell+1}^{N-1}\right)= \\
=\sum_{\operatorname{part}} \sum_{i=1}^{\ell} \sum_{j=l+1}^{N} \frac{\mathrm{T}_{i, j}(z)}{\lambda_{\ell}(z)} \frac{\left.\mathbb{B}\left(\left\{\bar{t}^{s}\right\}_{1}^{i-1},\left\{\bar{t}_{\mathrm{I}}^{s}\right\}_{i}^{\ell-1}, \bar{t}^{\ell},\left\{\bar{t}_{\mathrm{I}}^{s}\right\}_{\ell+1}^{j-1},\left\{\bar{t}^{s}\right\}_{j}^{N-1}\right)\right)}{f\left(z, \bar{t}^{\ell-1}\right) f\left(\bar{t}^{\ell+1}, z\right)} \times \\
\quad \times \prod_{p=i}^{\ell-1} \frac{\beta_{p}\left(\bar{t}_{\mathrm{I}}^{p}\right) \tilde{g}\left(\bar{t}_{\mathrm{I}}^{p+1}, \bar{t}_{\mathrm{I}}^{p}\right) f\left(\bar{t}_{\mathrm{I}}^{p}, \bar{t}_{\mathrm{II}}^{p}\right)}{f\left(\bar{t}_{\mathrm{I}}^{p}, \bar{t}^{p-1}\right)} \prod_{p=\ell+1}^{j-1} \frac{g\left(\bar{t}_{\mathrm{II}}^{p}, \bar{t}_{\mathrm{II}}^{p-1}\right) f\left(\bar{t}_{\mathrm{II}}^{p}, \bar{t}_{\mathrm{II}}^{p}\right)}{f\left(\bar{t}^{p+1}, \bar{t}_{\mathrm{II}}^{p}\right)},
\end{aligned}
$$


where sum goes over partitions $\left\{\bar{t}_{\mathrm{I}}^{p}, \bar{t}_{\mathrm{II}}^{p}\right\} \vdash \bar{t}^{p}$ for $p=i, \ldots, \ell-1$ and partitions $\left\{\bar{t}_{\mathrm{II}}^{p}, \bar{t}_{\mathrm{II}}^{p}\right\} \vdash$ $\bar{t}^{p}$ for $p=\ell+1, \ldots, j-1$ such that $\left|\bar{t}_{\mathrm{I}}^{p}\right|=1$ for $p=i, \ldots, \ell$ and $\left|\bar{t}_{\mathrm{II}}^{p}\right|=1$ for $p=$ $\ell, \ldots, j-1$ with fixed boundary partitions $\bar{t}_{\mathrm{I}}^{\ell}=\bar{t}_{\mathrm{II}}^{\ell}=\{z\}$ and $\bar{t}^{0}=\bar{t}^{N}=\varnothing$.

Proof of the proposition 3.4 can be found in the appendix B.

Recurrence relation (3.18) is written in assumption that all sets of the Bethe parameters $\bar{t}^{p}$ for $p=1, \ldots, N-1$ are not empty. If $\bar{t}^{\prime}=\varnothing$ for some $\ell^{\prime} \neq \ell$ then for $\ell^{\prime}>\ell$ the sum in (3.18) over $j$ ends at $j=\ell^{\prime}$ and for $\ell^{\prime}<\ell$ the the sum over $i$ begins at $\ell^{\prime}+1$.

Corollary 3.5. There are two extreme cases of the recurrence relations (3.18) with respect to first and last Bethe parameters when $\ell=1$ and $\ell=N-1$

$$
\begin{aligned}
\mathbb{B}\left(\left\{z, \bar{t}^{1}\right\} ;\left\{\bar{t}^{s}\right\}_{2}^{N-1}\right)= & \sum_{j=2}^{N} \frac{\mathrm{T}_{1, j}(z)}{\lambda_{1}(z)} \sum_{\mathrm{part}} \mathbb{B}\left(\bar{t}^{1} ;\left\{\bar{t}_{\mathrm{II}}^{s}\right\}_{2}^{j-1} ;\left\{\bar{t}^{s}\right\}_{j}^{N-1}\right) \times \\
& \times \frac{1}{f\left(\bar{t}^{2}, z\right)} \prod_{p=2}^{j-1} \frac{g\left(\bar{t}_{\mathrm{II}}^{p}, \bar{t}_{\mathrm{II}}^{p-1}\right) f\left(\bar{t}_{\mathrm{II}}^{p}, \bar{t}_{\mathrm{II}}^{p}\right)}{f\left(\bar{t}^{p+1}, \bar{t}_{\mathrm{II}}^{p}\right)}
\end{aligned}
$$

and

$$
\begin{aligned}
\mathbb{B}\left(\left\{\bar{t}^{s}\right\}_{1}^{N-2} ;\left\{z, \bar{t}^{N-1}\right\}\right)= & \sum_{j=1}^{N-1} \frac{\mathrm{T}_{j, N}(z)}{\lambda_{N-1}(z)} \sum_{\text {part }} \mathbb{B}\left(\left\{\bar{t}^{s}\right\}_{1}^{j-1} ;\left\{\bar{t}_{\mathrm{II}}^{s}\right\}_{j}^{N-2} ; \bar{t}^{N-1}\right) \times \\
& \times \frac{1}{f\left(z, \bar{t}^{N-2}\right)} \prod_{p=j}^{N-2} \frac{\beta_{p}\left(\bar{t}_{\mathrm{I}}^{p}\right) \tilde{g}\left(\bar{t}_{\mathrm{I}}^{p+1}, \bar{t}_{\mathrm{I}}^{p}\right) f\left(\bar{t}_{\mathrm{I}}^{p}, \bar{t}_{\mathrm{II}}^{p}\right)}{f\left(\bar{t}_{\mathrm{I}}^{p}, \bar{t}^{p-1}\right)}
\end{aligned}
$$

Recurrence relations (3.19) and (3.20) were obtained in [5] in the framework of the hierarchical Bethe ansatz techniques and for different normalization of the off-shell Bethe vectors described in appendix $\mathrm{B}$. One can verify that these recurrence relations are compatible with the hierarchical relations for the Bethe vectors described in [6].

\section{Zero modes method}

In this paper we present trigonometric version of the zero modes method developed for rational case in [4]. This method uses the fact that all entries of the monodromy matrix can be obtained from one selected entry using commutation relations with zero modes similar to (4.1) and (4.2). Using the action of zero modes and any particular element of monodromy matrix onto Bethe vector one can obtain an action of the rest entries by induction.

The induction step is based on relations between the action of monodromy matrix entry $\mathrm{T}_{i, j}(z)$ onto off-shell Bethe vector and the action of the entries $\mathrm{T}_{i+1, j}(z), \mathrm{T}_{i, j-1}(z)$ and zero modes $\mathrm{T}_{i+1, i}^{-}[0], \mathrm{T}_{j, j-1}^{-}[0]$ (see formulas (4.1) and (4.2) below). These relations allow to obtain the action of the entries $\mathrm{T}_{i+1, j}(z), \mathrm{T}_{i, j-1}(z)$ assuming the action of $\mathrm{T}_{i, j}(z)$. Since the action of the matrix entry $\mathrm{T}_{1, N}(z)$ can be calculated using explicit

presentation of the off-shell Bethe vector $\mathbb{B}(\bar{t})(3.8)$ one can prove by induction the 
action formulas of all entries $\mathrm{T}_{i, j}(z)$ for all $1 \leq i, j \leq N$. This is done in appendix $\mathrm{A}$. The action of the zero mode operators $\mathrm{T}_{\ell+1, \ell}^{-}[0]$ onto off-shell Bethe vectors are given by the proposition 4.1 and the action of the entry $\mathrm{T}_{1, N}(z)$ is calculated in proposition 4.2 .

To develop zero modes method one needs to use the commutation relations of the zero modes $\mathrm{T}_{l+1, l}^{-}[0]$ with monodromy matrix entry $\mathrm{T}_{i, j}(z) \equiv \mathrm{T}_{i, j}^{+}(z)$ for $l=i$ and $l=j-1$. Taking $v=0$ in (2.8) for $\mu=+, \nu=-$ and $\{i, j, k, l\} \rightarrow\{i, j, i+1, i\}$ one obtains

$$
\begin{aligned}
& \mathrm{T}_{i, j}(z) \mathrm{T}_{i+1, i}^{-}[0]-q^{\delta_{i, j}} \mathrm{~T}_{i+1, i}^{-}[0] \mathrm{T}_{i, j}(z)= \\
& \quad=\left(q-q^{-1}\right)\left(\delta_{i, j-1} \mathrm{~T}_{j, j}^{-}[0] \mathrm{T}_{i, i}(z)-\mathrm{T}_{i+1, j}(z) \mathrm{T}_{i, i}^{-}[0]\right) .
\end{aligned}
$$

Analogously, taking $u=0$ in the commutation relation (2.8) for $\mu=-, \nu=+$ and $\{i, j, k, l\} \rightarrow\{i, j, j, j-1\}$ one gets

$$
\begin{aligned}
& q^{-\delta_{i, j}} \mathrm{~T}_{j, j-1}^{-}[0] \mathrm{T}_{i, j}(z)-\mathrm{T}_{i, j}(z) \mathrm{T}_{j, j-1}^{-}[0]= \\
& \quad=\left(q-q^{-1}\right)\left(\delta_{i, j-1} \mathrm{~T}_{i, i}^{-}[0] \mathrm{T}_{j, j}(z)-\mathrm{T}_{i, j-1}(z) \mathrm{T}_{j, j}^{-}[0]\right)
\end{aligned}
$$

Also we will use commutation relation with zero modes of diagonal entries of monodromy matrices. Taking $v=0$ in (2.8) for $\mu=+, \nu=-$ and $\{i, j, k, l\} \rightarrow\{i, j, l, l\}$ one obtains

$$
q^{\delta_{i l}} \mathrm{~T}_{i, j}(z) \mathrm{T}_{l, l}^{-}[0]=q^{\delta_{l j}} \mathrm{~T}_{l, l}^{-}[0] \mathrm{T}_{i, j}(z)
$$

According to the Gauss decomposition (2.10) and restrictions (2.4)

$$
\mathrm{T}_{i, i}^{-}[0]=k_{i}^{-1}, \quad \mathrm{~T}_{i+1, i}^{-}[0]=k_{i}^{-1} E_{i}
$$

where $k_{i}$ and $E_{i}$ are zero modes operators of the Gauss coordinates $k_{i}^{+}(u)$ and $\mathrm{E}_{i, i+1}^{-}(u)$ respectively: $k_{i}=k_{i}^{+}[0]=\left.k_{i}^{+}(u)\right|_{u=\infty}$ and $E_{i}=\mathrm{E}_{i, i+1}^{-}[0]=\left.\mathrm{E}_{i, i+1}^{-}(u)\right|_{u=0}$.

The action of the zero mode operators onto vacuum vector $|\mathrm{vac}\rangle$ is defined as follows

$$
k_{i}|\mathrm{vac}\rangle=\kappa_{i}^{-1}|\mathrm{vac}\rangle, \quad E_{i}|\mathrm{vac}\rangle=0,
$$

where $\kappa_{i} \in \mathbb{C}$ are complex parameters .

The action of zero mode operators $\mathrm{T}_{i+1, i}^{-}[0]$ onto off-shell Bethe vector (3.8) is given by the following

\section{Proposition 4.1.}

$$
\begin{aligned}
& \mathrm{T}_{i+1, i}^{-}[0] \mathbb{B}(\bar{t})=\left(q-q^{-1}\right) \times \\
& \times \sum_{\ell=1}^{r_{i}}\left(\kappa_{i} q^{r_{i}-r_{i-1}-1} \beta_{i}\left(t_{\ell}^{i}\right) \frac{f\left(t_{\ell}^{i}, \bar{t}_{\ell}^{i}\right)}{f\left(t_{\ell}^{i}, \bar{t}^{i-1}\right)}-\kappa_{i+1} q^{r_{i+1}-r_{i}+1} \frac{f\left(\bar{t}_{\ell}^{i}, t_{\ell}^{i}\right)}{f\left(\bar{t}^{i+1}, \bar{t}_{\ell}^{i}\right)}\right) \mathbb{B}\left(\bar{t} \backslash\left\{t_{\ell}^{i}\right\}\right) .
\end{aligned}
$$

Proof. To prove this proposition one can use the presentation of the off-shell pre-Bethe vector 15

$$
P_{f}^{+}(\mathbb{F}(\bar{t}))=\mathbb{F}(\bar{t})+\sum_{i=1}^{N-1} \sum_{\ell=1}^{r_{i}} \frac{f\left(\bar{t}_{\ell}^{i}, t_{\ell}^{i}\right)}{f\left(\bar{t}^{i+1}, t_{\ell}^{i}\right)} \mathrm{F}_{i+1, i}^{-}\left(t_{\ell}^{i}\right) \mathbb{F}\left(\bar{t} \backslash\left\{t_{\ell}^{i}\right\}\right)+\cdots
$$


where $\cdots$ stands for the terms which are annihilated by projection $P_{f}^{+}$after adjoint action of zero mode operators $E_{i}, i=1, \ldots, N-1$. The commutation relations between Gauss coordinates for $\mu, \nu= \pm$

$$
\left[\mathrm{E}_{i, i+1}^{\mu}(u), \mathrm{F}_{j+1, j}^{\nu}(v)\right]=\delta_{i, j} g(v, u)\left(k_{i+1}^{\nu}(v) k_{i}^{\nu}(v)^{-1}-k_{i+1}^{\mu}(u) k_{i}^{\mu}(u)^{-1}\right)
$$

imply the commutation relations

$$
\left[E_{i}, \mathrm{~F}_{i+1, i}^{-}(v)\right]=\left(q-q^{-1}\right)\left(k_{i+1}^{-}(v) k_{i}^{-}(v)^{-1}-k_{i+1}^{-1} k_{i}\right)
$$

and

$$
\left[E_{i}, F_{i}(v)\right]=\left(q-q^{-1}\right)\left(k_{i+1}^{+}(v) k_{i}^{+}(v)^{-1}-k_{i+1}^{-}(v) k_{i}^{-}(v)^{-1}\right) .
$$

Formulas (4.3) yield

$$
k_{i} F_{i}(v) k_{i}^{-1}=q^{-1} F_{i}(v), \quad k_{i+1} F_{i}(v) k_{i+1}^{-1}=q F_{i}(v)
$$

which imply

$$
k_{i}^{-1} \mathbb{F}(\bar{t}) k_{i}=q^{r_{i}-r_{i-1}} \mathbb{F}(\bar{t}) .
$$

Equality (4.10) together with (2.14) yields

$$
\left[E_{i}, \mathbb{F}(\bar{t})\right]=\left(q-q^{-1}\right) \sum_{\ell=1}^{r_{i}} \frac{f\left(t_{\ell}^{i}, \bar{t}_{\ell}^{i}\right)}{f\left(t_{\ell}^{i}, \bar{t}^{i-1}\right)} \mathbb{F}\left(\bar{t} \backslash\left\{t_{\ell}^{i}\right\}\right) k_{i+1}^{+}\left(t_{\ell}^{i}\right) k_{i}^{+}\left(t_{\ell}^{i}\right)^{-1}
$$

where we skip the terms proportional to $k_{i+1}^{-}\left(t_{\ell}^{i}\right) k_{i}^{-}\left(t_{\ell}^{i}\right)^{-1}$ since they will vanish after restriction of the action $\mathrm{T}_{i+1, i}^{-}[0] P_{f}^{+}(\mathbb{F}(\bar{t}))$ onto subalgebra $U_{F}^{+}=U_{F} \cap U_{q}^{+}\left(\tilde{\mathfrak{g l}}_{N}\right)$. Taking into account (4.4), (4.5), (4.7), (4.9) and (4.12) we obtain the statement of the proposition.

Remark 4.1. Note that unlike the rational quantum integrable models the on-shell Bethe vectors are not the highest weight vectors of $U_{q}\left(\mathfrak{g l}_{N}\right)$ with respect to the action of $E_{i}$.

In proving the action formulas of the monodromy matrix entries onto off-shell Bethe vectors we will use induction to obtain the action of the entries $T_{i+1, j}(z)$ and $T_{i, j-1}(z)$ from the induction assumption of the action $T_{i, j}(z)$. To do this we will equate the terms of (4.1) and (4.2) acting onto $\mathbb{B}(\bar{t})$ at the different parameters $\kappa_{i}$ using their arbitrariness. In order to formulate the base of the induction one can prove the following

Proposition 4.2. The monodromy matrix element $\mathrm{T}_{1, N}(z)$ is acting onto off-shell Bethe vector $\mathbb{B}(\bar{t})$ as follows

$$
\mathrm{T}_{1, N}(z) \mathbb{B}(\bar{t})=\lambda_{1}(z) \mathbb{B}(\bar{w})
$$

where $\bar{w}$ is a collection of sets of the variables $\left\{\bar{w}^{1}, \ldots, \bar{w}^{N-1}\right\}$ such that $\bar{w}^{i}=\left\{z, \bar{t}^{i}\right\}$ for $i=1, \ldots, N-1$.

Proof. To prove this proposition we need an auxiliary 
Lemma 4.3. There are the equalities in $U_{q}\left(\tilde{\mathfrak{g l}}_{N}\right)$

$$
\mathrm{F}_{N, 1}^{+}(z)=P_{f}^{+}\left(F_{N-1}(z) F_{N-2}(z) \cdots F_{2}(z) F_{1}(z)\right)
$$

and

$$
P_{f}^{+}\left(\mathrm{T}_{1, N}(z) \cdot \mathrm{F}_{j, i}^{-}(t)\right)=P_{f}^{+}\left(\mathrm{F}_{N, 1}^{+}(z) k_{1}^{+}(z) \cdot \mathrm{F}_{j, i}^{-}(t)\right)=0, \quad \forall i, j .
$$

Proof. The statement of the lemma can be proved using RTT relation (2.8) (see appendix D in [13] for details).

To calculate the action (4.14) we will use (4.15) and (4.16)

$$
\begin{aligned}
& \mathrm{T}_{1, N}(z) \cdot \mathbb{B}(\bar{t})=\mathrm{T}_{1, N}(z) \cdot P_{f}^{+}(\mathbb{F}(\bar{t}))|\operatorname{vac}\rangle=P_{f}^{+}\left(\mathrm{T}_{1, N}(z) \cdot \mathbb{F}(\bar{t})\right)|\operatorname{vac}\rangle= \\
& \quad=\left.P_{f}^{+}\left(F_{N-1}\left(z_{N-1}\right) \cdots F_{1}\left(z_{1}\right) k_{1}^{+}\left(z_{1}\right) \mathbb{F}(\bar{t})\right)|\operatorname{vac}\rangle\right|_{z_{i}=z}=\lambda_{1}(z) X(z, \bar{t}) \mathbb{B}(\bar{w}),
\end{aligned}
$$

where

$$
X(z, \bar{t})=\left.f\left(\bar{t}^{1}, z_{1}\right) \prod_{i=1}^{N-1} \frac{\gamma_{f}\left(\bar{t}^{i}\right)}{\gamma_{f}\left(\bar{w}^{i}\right)} \prod_{i=1}^{N-2} \frac{f\left(\bar{w}^{i+1}, \bar{w}^{i}\right)}{f\left(\bar{t}^{i+1}, \bar{t}^{i}\right) f\left(z_{i+1}, \bar{w}^{i}\right)}\right|_{z_{i}=z}=1
$$

and for $i=1, \ldots, N-1$

$$
\gamma_{f}\left(\bar{t}^{i}\right)=\prod_{1 \leq \ell<\ell^{\prime} \leq\left|\bar{t}^{i}\right|} f\left(t_{\ell^{\prime}}^{i}, t_{\ell}^{i}\right) .
$$

In order to perform this calculation using the commutation relations between total currents (2.14) we split parameters $z_{i} \neq z_{j}$ and denote $\bar{w}^{i}=\left\{z_{i}, \bar{t}^{i}\right\}$. In (4.17) we also use property of the projections that [12]

$$
P_{f}^{+}\left(\mathrm{F}_{N, 1}^{+}(z) \mathbb{F}(\bar{t})\right)=P_{f}^{+}\left(P_{f}^{+}\left(F_{N-1}(z) \cdots F_{1}(z)\right) \mathbb{F}(\bar{t})\right)=P_{f}^{+}\left(F_{N-1}(z) \cdots F_{1}(z) \mathbb{F}(\bar{t})\right) .
$$

\section{Dual Bethe vector and scalar product}

Definition of dual Bethe vectors $\mathbb{C}(\bar{u})$ for $U_{q}\left(\tilde{\mathfrak{g l}}_{N}\right)$ and scalar product between $\mathbb{C}(\bar{u})$ and $\mathbb{B}(\bar{t})$ was given in [5]. The recurrence relations for the highest coefficient (HC) of the scalar product corresponding to the extreme recurrence relations (3.19) and (3.20) were found in this paper. Here we extend this result to more general recurrence relation (3.18) given by the proposition 3.4.

Dual Bethe vectors are the vectors of the dual representation space $\mathcal{H}^{*}$ of the algebra $U_{q}\left(\tilde{\mathfrak{g l}}_{N}\right)$ which possesses a vector $\langle$ vac| with properties similar to (2.9)

$$
\langle\operatorname{vac}| \mathrm{T}_{i, j}(u)=0, \quad i<j, \quad\langle\operatorname{vac}| \mathrm{T}_{i, i}(u)=\lambda_{i}(u)\langle\operatorname{vac}| .
$$

In order to define dual Bethe vectors we consider an involutive antimorphism

$$
\Psi: U_{q}\left(\tilde{\mathfrak{g l}}_{N}\right) \rightarrow U_{q^{-1}}\left(\tilde{\mathfrak{g l}}_{N}\right), \quad \Psi(A \cdot B)=\Psi(B) \cdot \Psi(A)
$$

defined on the matrix entries of the T-operators as follows

$$
\Psi\left(\mathrm{T}_{i, j}(u)\right)=\tilde{\mathrm{T}}_{j, i}\left(u^{-1}\right)
$$


where $\tilde{\mathrm{T}}_{i, j}(u) \in U_{q^{-1}}\left(\tilde{\mathfrak{g l}}_{N}\right)$.

The action of antimorphism (5.2) can be extended to the action onto vectors from representation spaces $\mathcal{H}$ and $\mathcal{H}^{*}$ according to the rules

$$
\begin{array}{ll}
\Psi(|\operatorname{vac}\rangle)=\langle\tilde{\operatorname{vac}}|, \quad & \Psi(A|\operatorname{vac}\rangle)=\langle\tilde{\operatorname{vac}}| \Psi(A), \\
\Psi(\langle\operatorname{vac}|)=|\tilde{\operatorname{vac}}\rangle, & \Psi(\langle\operatorname{vac}| A)=\Psi(A)|\tilde{\operatorname{vac}}\rangle .
\end{array}
$$

Here vectors $|\tilde{v a c}\rangle$ and $\langle\tilde{v a c}|$ are defined by (2.9) and (5.1) for the algebra $U_{q^{-1}}\left(\tilde{\mathfrak{g l}}_{N}\right)$ and $A$ is any product of the entries $\mathrm{T}_{i, j}(z)$ of the T-operator. Note also that according to the equalities

$$
\Psi\left(\mathrm{T}_{i, i}(z)|\operatorname{vac}\rangle\right)=\lambda_{i}(z)\langle\tilde{\operatorname{vac}}|=\langle\tilde{\operatorname{vac}}| \tilde{\mathrm{T}}_{i, i}\left(z^{-1}\right)=\tilde{\lambda}_{i}\left(z^{-1}\right)\langle\tilde{\operatorname{vac}}|
$$

one has

$$
\tilde{\lambda}_{i}\left(z^{-1}\right)=\lambda_{i}(z) .
$$

Here $\tilde{\lambda}_{i}(z)$ are eigenvalues of the diagonal entries of the T-operator for the algebra $U_{q^{-1}}\left(\tilde{g l l}_{N}\right)$.

It was demonstrated in [5] that the dual Bethe vectors can be defined as follows

$$
\Psi\left(\mathbb{B}_{q}(\bar{t})\right)=\mathbb{C}_{q^{-1}}\left(\bar{t}^{-1}\right), \quad \Psi\left(\mathbb{C}_{q}(\bar{t})\right)=\mathbb{B}_{q^{-1}}\left(\bar{t}^{-1}\right),
$$

where notation $\bar{t}^{-1}$ means

$$
\bar{t}^{-1}=\left\{\frac{1}{t_{1}^{1}}, \ldots, \frac{1}{t_{r_{1}}^{1}} ; \frac{1}{t_{1}^{2}}, \ldots, \frac{1}{t_{r_{2}}^{2}} ; \ldots ; \frac{1}{t_{1}^{N-1}}, \ldots, \frac{1}{t_{r_{N-1}-1}^{N-1}}\right\}
$$

and subscript in notation of the Bethe vectors $\mathbb{B}_{q^{-1}}(\bar{t})$ and $\mathbb{C}_{q^{-1}}(\bar{t})$ signifies that they are constructed for the algebra $U_{q^{-1}}\left(\tilde{\mathfrak{g l}}_{N}\right)$.

The scalar product of a generic Bethe vector $\mathbb{B}(\bar{t})$ and a generic dual Bethe vector $\mathbb{C}(\bar{u})$

$$
S(\bar{u} \mid \bar{t})=\mathbb{C}(\bar{u}) \mathbb{B}(\bar{t})
$$

is not vanishing unless $\left|\bar{u}^{i}\right|=\left|\bar{t}^{i}\right|$ for all $i=1, \ldots, N-1$. The scalar product $S(\bar{u} \mid \bar{t})$ can be written in the form [5]

$$
S(\bar{u} \mid \bar{t})=\sum_{\text {part }} W\left(\bar{u}_{\mathrm{I}}, \bar{u}_{\mathrm{I}} \mid \bar{t}_{\mathrm{I}}, \bar{t}_{\mathrm{II}}\right) \prod_{i=1}^{N-1} \beta_{i}\left(\bar{u}_{\mathrm{II}}^{i}\right) \beta_{i}\left(\bar{t}_{\mathrm{I}}^{i}\right),
$$

where sum goes over partitions $\left\{\bar{u}_{\mathrm{I}}^{i}, \bar{u}_{\mathrm{II}}^{i}\right\} \vdash \bar{u}^{i}$ and $\left\{\bar{t}_{\mathrm{I}}^{i}, \bar{t}_{\mathrm{II}}^{i}\right\} \vdash \bar{t}^{i}$ such that $\left|\bar{u}_{\mathrm{I}}^{i}\right|=\left|\bar{t}_{\mathrm{I}}^{i}\right|$ for $i=1, \ldots, N-1$. The rational function $W\left(\bar{u}_{\mathrm{I}}, \bar{u}_{\mathrm{I}} \mid \bar{t}_{\mathrm{I}}, \bar{t}_{\mathrm{II}}\right)$ depends only on the R-matrix of the model and does not depend on the free functional parameters $\lambda_{i}(z)$. The function $W\left(\bar{u}_{\mathrm{I}}, \bar{u}_{\mathrm{II}} \mid \bar{t}_{\mathrm{I}}, \bar{t}_{\mathrm{II}}\right)$ has a presentation [5]

$$
W\left(\bar{u}_{\mathrm{I}}, \bar{u}_{\mathrm{II}} \mid \bar{t}_{\mathrm{I}}, \bar{t}_{\mathrm{II}}\right)=Z\left(\bar{u}_{\mathrm{I}} \mid \bar{t}_{\mathrm{I}}\right) \bar{Z}\left(\bar{u}_{\mathrm{II}} \mid \bar{t}_{\mathrm{II}}\right) \frac{\prod_{i=1}^{N-1} f\left(\bar{u}_{\mathrm{II}}^{i}, \bar{u}_{\mathrm{I}}^{i}\right) f\left(\bar{t}_{\mathrm{I}}^{i}, \bar{t}_{\mathrm{II}}^{i}\right)}{\prod_{i=1}^{N-2} f\left(\bar{u}_{\mathrm{II}}^{i+1}, \bar{u}_{\mathrm{I}}^{i}\right) f\left(\bar{t}_{\mathrm{I}}^{i+1}, \bar{t}_{\mathrm{II}}^{i}\right)},
$$

where

$$
W(\bar{u}, \varnothing \mid \bar{t}, \varnothing)=Z(\bar{u} \mid \bar{t}), \quad W(\varnothing, \bar{u} \mid \varnothing, \bar{t})=\bar{Z}(\bar{u} \mid \bar{t})
$$


are so called highest coefficient of the scalar product. One can check that $\bar{Z}(\bar{u} \mid \bar{t})=$ $Z(\bar{t} \mid \bar{u})$ due to the symmetry $S(\bar{u} \mid \bar{t})=\Psi(S(\bar{u} \mid \bar{t}))=S(\bar{t} \mid \bar{u})$.

Recurrence relation (3.18) implies the recurrence relation for the dual Bethe vectors

$$
\begin{aligned}
\mathbb{C}\left(\left\{\bar{u}^{s}\right\}_{1}^{\ell-1},\left\{\bar{u}_{\mathrm{I}}^{\ell}, \bar{u}_{\mathrm{II}}^{\ell}\right\}\right. & \left.,\left\{\bar{u}^{s}\right\}_{\ell+1}^{N-1}\right)=\sum_{i=1}^{\ell} \sum_{j=\ell+1}^{N} \sum_{\text {part }} \frac{\left.\mathbb{C}\left(\left\{\bar{u}^{s}\right\}_{1}^{i-1},\left\{\bar{u}_{\mathrm{II}}^{s}\right\}_{i}^{j-1},\left\{\bar{u}^{s}\right\}_{j}^{N-1}\right)\right)}{f\left(\bar{u}_{\mathrm{I}}^{\ell}, \bar{u}^{\ell-1}\right) f\left(\bar{u}^{\ell+1}, \bar{u}_{\mathrm{I}}^{\ell}\right)} \frac{T_{j, i}\left(\bar{u}_{\mathrm{I}}^{\ell}\right)}{\lambda_{\ell}\left(\bar{u}_{\mathrm{I}}^{\ell}\right)} \times \\
& \times \prod_{p=i}^{\ell-1} \frac{\beta_{p}\left(\bar{u}_{\mathrm{I}}^{p}\right) g\left(\bar{u}_{\mathrm{I}}^{p+1}, \bar{u}_{\mathrm{I}}^{p}\right) f\left(\bar{u}_{\mathrm{I}}^{p}, \bar{u}_{\mathrm{II}}^{p}\right)}{f\left(\bar{u}_{\mathrm{I}}^{p}, \bar{u}^{p-1}\right)} \prod_{p=\ell+1}^{j-1} \frac{\tilde{g}\left(\bar{u}_{\mathrm{II}}^{p}, \bar{u}_{\mathrm{II}}^{p-1}\right) f\left(\bar{u}_{\mathrm{II}}^{p}, \bar{u}_{\mathrm{II}}^{p}\right)}{f\left(\bar{u}^{p+1}, \bar{u}_{\mathrm{II}}^{p}\right)}
\end{aligned}
$$

where we used (5.5),

$$
f_{q^{-1}}\left(x^{-1}, y^{-1}\right)=f_{q}(x, y), \quad g_{q^{-1}}\left(x^{-1}, y^{-1}\right)=\tilde{g}_{q}(x, y)
$$

and sum over partitions is the same as in (3.18). Note that there are no summation over partition of the set $\bar{u}^{\ell}$ in (5.11).

Let us consider the scalar product (5.7) for some fixed partition $\left\{\bar{u}_{\mathrm{I}}^{\ell}, \bar{u}_{\mathrm{II}}^{\ell}\right\} \vdash \bar{u}^{\ell}$ with cardinality $\left|\bar{u}_{\mathrm{I}}^{\ell}\right|=1$. Then, one can prove following

Proposition 5.1. Highest coefficients $Z(\bar{u} \mid \bar{t})$ and $\bar{Z}(\bar{u} \mid \bar{t})$ satisfy the recurrence relations

$$
\begin{aligned}
Z(\bar{u} \mid \bar{t})=\sum_{\text {part }} \frac{f\left(\bar{t}^{\ell}, \bar{u}_{\mathrm{I}}^{\ell}\right)}{f\left(\bar{u}_{\mathrm{I}}^{\ell}, \bar{u}^{\ell-1}\right) f\left(\bar{u}^{\ell+1}, \bar{u}_{\mathrm{I}}^{\ell}\right)} \frac{g\left(\bar{t}_{\mathrm{I}}^{\ell}, \bar{w}_{\mathrm{I}}^{\ell-1}\right) f\left(\bar{t}_{\mathrm{I}}^{\ell}, \bar{t}_{\mathrm{II}}^{\ell}\right)}{f\left(\bar{t}_{\mathrm{I}}^{\ell}, \bar{w}^{\ell-1}\right)} \\
\times \sum_{j=\ell+1}^{N} \prod_{p=1}^{\ell-1} \frac{g\left(\bar{w}_{\mathrm{I}}^{p}, \bar{w}_{\mathrm{I}}^{p-1}\right) f\left(\bar{w}_{\mathrm{I}}^{p}, \bar{w}_{\mathrm{II}}^{p}\right)}{f\left(\bar{w}_{\mathrm{I}}^{p}, \bar{w}^{p-1}\right)} \prod_{p=\ell+1}^{j-1} \frac{\tilde{g}\left(\bar{u}_{\mathrm{II}}^{p}, \bar{u}_{\mathrm{II}}^{p-1}\right) f\left(\bar{u}_{\mathrm{II}}^{p}, \bar{u}_{\mathrm{II}}^{p}\right)}{f\left(\bar{u}^{p+1}, \bar{u}_{\mathrm{II}}^{p}\right)} \frac{g\left(\bar{t}_{\mathrm{I}}^{p}, \bar{t}_{\mathrm{I}}^{p-1}\right) f\left(\bar{t}_{\mathrm{I}}^{p}, \bar{t}_{\mathrm{II}}^{p}\right)}{f\left(\bar{t}_{\mathrm{I}}^{p}, \bar{t}^{p-1}\right)} \\
\quad \times Z\left(\left\{\bar{u}^{s}\right\}_{1}^{\ell-1},\left\{\bar{u}_{\mathrm{II}}^{s}\right\}_{\ell}^{j-1},\left\{\bar{u}^{s}\right\}_{j}^{N-1} \mid\left\{\bar{w}_{\mathrm{II}}^{s}\right\}_{1}^{\ell-1},\left\{\bar{t}_{\mathrm{II}}^{s}\right\}_{\ell}^{j-1},\left\{\bar{t}^{s}\right\}_{j}^{N-1}\right)
\end{aligned}
$$

and

$$
\begin{gathered}
\bar{Z}(\bar{u} \mid \bar{t})=\sum_{\text {part }} \frac{f\left(\bar{u}_{\mathrm{I}}^{\ell}, \bar{t}^{\ell}\right)}{f\left(\bar{u}_{\mathrm{I}}^{\ell}, \bar{u}^{\ell-1}\right) f\left(\bar{u}^{\ell+1}, \bar{u}_{\mathrm{I}}^{\ell}\right)} \frac{\tilde{g}\left(\bar{w}_{\mathrm{II}}^{\ell+1}, \bar{t}_{\mathrm{II}}^{\ell}\right) f\left(\bar{t}_{\mathrm{II}}^{\ell}, \bar{t}_{\mathrm{II}}^{\ell}\right)}{f\left(\bar{w}^{\ell+1}, \bar{t}_{\mathrm{II}}^{\ell}\right)} \\
\times \sum_{i=1}^{\ell} \prod_{p=i}^{\ell-1} \frac{g\left(\bar{u}_{\mathrm{I}}^{p+1}, \bar{u}_{\mathrm{I}}^{p}\right) f\left(\bar{u}_{\mathrm{I}}^{p}, \bar{u}_{\mathrm{II}}^{p}\right)}{f\left(\bar{u}_{\mathrm{I}}^{p}, \bar{u}^{p-1}\right)} \frac{\tilde{g}\left(\bar{t}_{\mathrm{II}}^{p+1}, \bar{t}_{\mathrm{II}}^{p}\right) f\left(\bar{t}_{\mathrm{II}}^{p}, \bar{t}_{\mathrm{II}}^{p}\right)}{f\left(\bar{t}^{p+1}, \bar{t}_{\mathrm{III}}^{p}\right)} \prod_{p=\ell+1}^{N-1} \frac{\tilde{g}\left(\bar{w}_{\mathrm{II}}^{p+1}, \bar{w}_{\mathrm{II}}^{p}\right) f\left(\bar{w}_{\mathrm{II}}^{p}, \bar{w}_{\mathrm{III}}^{p}\right)}{f\left(\bar{w}^{p+1}, \bar{w}_{\mathrm{III}}^{p}\right)} \\
\times \bar{Z}\left(\left\{\bar{u}^{s}\right\}_{1}^{i-1},\left\{\bar{u}_{\mathrm{II}}^{s}\right\}_{i}^{\ell},\left\{\bar{u}^{s}\right\}_{\ell+1}^{N-1} \mid\left\{\bar{t}^{s}\right\}_{1}^{i-1},\left\{\bar{t}_{\mathrm{II}}^{s}\right\}_{i}^{\ell},\left\{\bar{w}_{\mathrm{II}}^{s}\right\}_{\ell+1}^{N-1}\right) .
\end{gathered}
$$

In (5.12) partition is going over $\left\{\bar{u}_{\mathrm{II}}^{s}, \bar{u}_{\mathrm{II}}^{s}\right\} \vdash \bar{u}^{s}$ for $\ell+1 \leq s \leq j-1,\left\{\bar{t}_{\mathrm{I}}^{s}, \bar{t}_{\mathrm{II}}^{s}\right\} \vdash \bar{t}^{s}$ for $\ell \leq s \leq j-1$ and $\left\{\bar{u}_{\mathrm{I}}^{\ell}, \bar{t}^{s}\right\}=\left\{\bar{w}_{\mathrm{I}}^{s}, \bar{w}_{\mathrm{II}}^{s}\right\} \vdash \bar{w}^{s}$ for $1 \leq s \leq \ell-1$. In (5.13) partition is going over $\left\{\bar{u}_{\mathrm{I}}^{s}, \bar{u}_{\mathrm{II}}^{s}\right\} \vdash \bar{u}^{s}$ for $i \leq s \leq \ell-1,\left\{\bar{t}_{\mathrm{II}}^{s}, \bar{t}_{\mathrm{II}}^{s}\right\} \vdash \bar{t}^{s}$ for $i \leq s \leq \ell$ and $\left\{\bar{u}_{\mathrm{I}}^{\ell}, \bar{t}^{s}\right\}=\left\{\bar{w}_{\mathrm{II}}^{s}, \bar{w}_{\mathrm{II}}^{s}\right\} \vdash \bar{w}^{s}$ for $\ell+1 \leq s \leq N-1$. In both formulas (5.12) and (5.13) there are no partition over set $\bar{u}^{\ell}$. Boundary sets are defined as follows: $\bar{u}^{0}=\bar{t}^{0}=\bar{w}_{\mathrm{II}}^{0}=\bar{u}^{N}=\bar{t}^{N}=\bar{w}_{\mathrm{II}}^{N}=\varnothing$ and $\bar{w}_{\mathrm{I}}^{0}=\bar{w}_{\mathrm{II}}^{N}=\bar{u}_{\mathrm{I}}^{\ell}$.

Proof of this proposition consists of the three steps. 
1. First, one substitutes recurrence relation for the dual Bethe vector (5.11) in the right hand side of the equality

$$
\mathbb{C}(\bar{u}) \mathbb{B}(\bar{t})=\mathbb{C}\left(\left\{\bar{u}^{s}\right\}_{1}^{\ell-1},\left\{\bar{u}_{\mathrm{I}}^{\ell}, \bar{u}_{\mathrm{I}}^{\ell}\right\},\left\{\bar{u}^{s}\right\}_{\ell+1}^{N-1}\right) \mathbb{B}(t)
$$

for some fixed partition $\left\{\bar{u}_{\mathrm{I}}^{\ell}, \bar{u}_{\mathrm{II}}^{\ell}\right\} \vdash \bar{u}^{\ell}$.

2. Then, one uses the action of the monodromy matrix entry $T_{j, i}\left(\bar{u}_{\mathrm{I}}^{\ell}\right)$ onto Bethe vector $\mathbb{B}(\bar{t})$ according to the proposition 3.2 .

3. Finally, the statement of the proposition follows from comparing the coefficients at the products $\prod_{i=1}^{N-1} \beta_{i}\left(\bar{t}^{i}\right)$ and $\prod_{i=1}^{N-1} \beta_{i}\left(\bar{u}^{i}\right)$ in both sides of (15.14) in the presentation (5.8).

The extreme case $\ell=1$ in (5.12) takes the form

$$
\begin{gathered}
Z(\bar{u} \mid \bar{t})=\sum_{\text {part }} \sum_{j=2}^{N} \frac{g\left(\bar{t}_{\mathrm{I}}^{1}, \bar{u}_{\mathrm{I}}^{1}\right) f\left(\bar{t}_{\mathrm{II}}, \bar{u}_{\mathrm{I}}^{1}\right) f\left(\bar{t}_{\mathrm{I}}^{1}, \bar{t}_{\mathrm{II}}^{1}\right)}{f\left(\bar{u}^{2}, \bar{u}_{\mathrm{I}}^{1}\right)} \prod_{p=2}^{j-1} \frac{\tilde{g}\left(\bar{u}_{\mathrm{II}}^{p}, \bar{u}_{\mathrm{II}}^{p-1}\right) f\left(\bar{u}_{\mathrm{I}}^{p}, \bar{u}_{\mathrm{III}}^{p}\right)}{f\left(\bar{u}^{p+1}, \bar{u}_{\mathrm{II}}^{p}\right)} \frac{g\left(\bar{t}_{\mathrm{I}}^{p}, \bar{t}_{\mathrm{I}}^{p-1}\right) f\left(\bar{t}_{\mathrm{I}}^{p}, \bar{t}_{\mathrm{II}}^{p}\right)}{f\left(\bar{t}_{\mathrm{I}}^{p}, \bar{t}^{p-1}\right)} \\
Z\left(\left\{\bar{u}_{\mathrm{II}}^{s}\right\}_{1}^{j-1},\left\{\bar{u}^{s}\right\}_{j}^{N-1} \mid\left\{\bar{t}_{\mathrm{II}}^{s}\right\}_{1}^{j-1},\left\{\bar{t}^{s}\right\}_{j}^{N-1}\right),
\end{gathered}
$$

where partition is going over $\left\{\bar{u}_{\mathrm{I}}^{s}, \bar{u}_{\mathrm{II}}^{s}\right\} \vdash \bar{u}^{s}$ for $2 \leq s \leq j-1,\left\{\bar{t}_{\mathrm{II}}^{s}, \bar{t}_{\mathrm{II}}^{s}\right\} \vdash \bar{t}^{s}$ for $i \leq 1 \leq$ $j-1$. One can check that this equality coincide identically with formula (4.15) from [5].

The extreme case $\ell=N-1$ in (5.13) takes the form

$$
\begin{aligned}
\bar{Z}(\bar{u} \mid \bar{t})=\sum_{i=1}^{N-1} \sum_{\text {part }} \frac{\tilde{g}\left(\bar{u}_{\mathrm{I}}^{N-1}, \bar{t}_{\mathrm{II}}^{N-1}\right) f\left(\bar{u}_{\mathrm{I}}^{N-1}, \bar{t}_{\mathrm{II}}^{N-1}\right) f\left(\bar{t}_{\mathrm{II}}^{N-1}, \bar{t}_{\mathrm{II}}^{N-1}\right)}{f\left(\bar{u}_{\mathrm{I}}^{N-1}, \bar{u}^{N-2}\right)} \\
\times \prod_{p=i}^{N-2} \frac{\tilde{g}\left(\bar{t}_{\mathrm{II}}^{p+1}, \bar{t}_{\mathrm{II}}^{p}\right) f\left(\bar{t}_{\mathrm{II}}^{p}, \bar{t}_{\mathrm{II}}^{p}\right)}{f\left(\bar{t}^{p+1}, \bar{t}_{\mathrm{II}}^{p}\right)} \frac{g\left(\bar{u}_{\mathrm{I}}^{p+1}, \bar{u}_{\mathrm{I}}^{p}\right) f\left(\bar{u}_{\mathrm{I}}^{p}, \bar{u}_{\mathrm{II}}^{p}\right)}{f\left(\bar{u}_{\mathrm{I}}^{p}, \bar{u}^{p-1}\right)} \\
\quad \times \bar{Z}\left(\left\{\bar{u}^{s}\right\}_{1}^{i-1},\left\{\bar{u}_{\mathrm{II}}^{s}\right\}_{i}^{N-1} \mid\left\{\bar{t}^{s}\right\}_{1}^{i-1},\left\{\bar{t}_{\mathrm{II}}^{s}\right\}_{i}^{N-1}\right),
\end{aligned}
$$

where partition is going over $\left\{\bar{u}_{\mathrm{I}}^{s}, \bar{u}_{\mathrm{II}}^{s}\right\} \vdash \bar{u}^{s}$ for $i \leq s \leq N-2,\left\{\bar{t}_{\mathrm{II}}^{s}, \bar{t}_{\mathrm{II}}^{s}\right\} \vdash \bar{t}^{s}$ for $i \leq s \leq N-1$. Again, equality (5.16) coincide identically with formula (4.16) from [5] if one takes into account equality $\bar{Z}(\bar{u} \mid \bar{t})=Z(\bar{t} \mid \bar{u})$.

\section{Conclusion}

This paper is a continuation of the research started in [4] to develop zero modes method for the quantum integrable models defined by $U_{q}\left(\mathfrak{g l}_{N}\right)$-invariant R-matrices. The aim of this method is to find the action formulas of monodromy matrix entries onto off-shell Bethe vectors. These action formulas can be further used to obtain various recurrence relations for the Bethe vectors itself and different physical quantities in this class of the quantum integrable models. In [4] the recurrence relations for the highest coefficients of the scalar products of the off-shell Bethe vectors were obtained for the rational integrable models associated to super-Yangian double $D Y(\mathfrak{g l}(m \mid n)$. In this paper we extend these results to the quantum integrable models based on the quantum loop algebra $U_{q}\left(\tilde{\mathfrak{g l}}_{N}\right)$. 


\section{Acknowledgments}

The work of SP was supported in part by the RFBR Grant 19-01-00726-a.

\section{A Proof of the proposition 3.2}

The statement of this proposition is obviously equivalent to (4.7) from the proposition 4.2 for $i=1$ and $j=N$. Assume that it is valid also for arbitrary values $i$ and $j$ and prove using (4.1) that it is valid as well for the action of $\mathrm{T}_{i+1, j}(z)$. We will consider only the case of $j \geq i+1$ in details, since the case $j<i+1$ can be considered analogously.

Due to

$$
\mathrm{T}_{i, i}^{-}[0] \mathbb{B}(\bar{t})=\kappa_{i} q^{r_{i}-r_{i-1}} \mathbb{B}(\bar{t}),
$$

the action of (4.1) onto off-shell Bethe vector $\mathbb{B}(\bar{t})$ transforms into

$$
\begin{aligned}
& \left(\mathrm{T}_{i+1, i}^{-}[0] \mathrm{T}_{i, j}(z)-\mathrm{T}_{i, j}(z) \mathrm{T}_{i+1, i}^{-}[0]\right) \mathbb{B}(\bar{t})= \\
& \quad=\left(q-q^{-1}\right)\left(q^{r_{i}-r_{i-1}} \kappa_{i} \mathrm{~T}_{i+1, j}(z) \mathbb{B}(\bar{t})-\delta_{i+1, j} q^{r_{i+1}-r_{i}} \kappa_{i+1} \mathrm{~T}_{i, i}(z) \mathbb{B}(\bar{t})\right)
\end{aligned}
$$

where we used commutativity $\left[\mathrm{T}_{i+1, i+1}^{-}[0], \mathrm{T}_{i, i}(z)\right]=0$. To obtain the action $\mathrm{T}_{i+1, j}(z)$ onto off-shell Bethe vector $\mathbb{B}(\bar{t})$ from $(\mathrm{A} .2)$ we equate the terms proportional to $\kappa_{i}$ and check that the terms proportional to $\kappa_{i+1}$ are cancel each other in the left hand side of (A.2) for $j>i+1$ and yields the action of $\mathrm{T}_{i, i}(z) \mathbb{B}(\bar{t})$ for $j=i+1$.

For $j \geq i+1$ in the action (3.15) $\bar{w}^{s}=\left\{z, \bar{t}^{s}\right\}=\bar{w}_{\mathrm{II}}^{s}$ and $\bar{w}_{\mathrm{I}}^{s}=\bar{w}_{\mathrm{III}}^{s}=\varnothing$ for $s=i, \ldots, j-1$. Using (4.6) one gets

$$
\begin{aligned}
\left.\mathrm{T}_{i, j}(z) \mathrm{T}_{i+1, i}^{-}[0] \mathbb{B}(\bar{t})\right|_{\kappa_{i+1}=0}= & \left(q-q^{-1}\right) \kappa_{i} q^{r_{i}-r_{i-1}-1} \lambda_{1}(z) \times \\
& \times \sum_{\ell=1}^{r_{i}} \beta_{i}\left(t_{\ell}^{i}\right) \frac{f\left(t_{\ell}^{i}, \bar{t}_{\ell}^{i}\right)}{f\left(t_{\ell}^{i}, \bar{t}^{i-1}\right)} \sum_{\text {part }} \mathbb{B}\left(\bar{w}_{\text {II }}\right) \mathcal{A}_{i, j}\left(\bar{w}_{\mathrm{I}} ; \bar{w}_{\mathrm{II}} ; \bar{w}_{\mathrm{II}}\right),
\end{aligned}
$$

where $\bar{w}_{\mathrm{II}}^{i}=\left\{z, \bar{t}_{\ell}^{i}\right\}$. Acting by the same operators in inverse order one gets

$$
\begin{aligned}
\left.\mathrm{T}_{i+1, i}^{-}[0] \mathrm{T}_{i, j}(z) \mathbb{B}(\bar{t})\right|_{\kappa_{i+1}=0} & =\left(q-q^{-1}\right) \kappa_{i} q^{r_{i}-r_{i-1}} \lambda_{1}(z) \times \\
& \times \sum_{\text {part }} \mathbb{B}\left(\bar{w}_{\mathrm{II}}\right) \beta_{i}\left(\bar{w}_{\mathrm{I}}^{i}\right) \frac{f\left(\bar{w}_{\mathrm{I}}^{i}, \bar{w}_{\mathrm{II}}^{i}\right)}{f\left(\bar{w}_{\mathrm{I}}^{i}, \bar{w}_{\mathrm{II}}^{i-1}\right)} \mathcal{A}_{i, j}\left(\bar{w}_{\mathrm{I}} ; \bar{w}_{\mathrm{II}} ; \bar{w}_{\mathrm{III}}\right),
\end{aligned}
$$

where the set $\bar{w}^{i}=\left\{z, \bar{t}^{i}\right\}$ is divided into subsets $\left\{\bar{w}_{\mathrm{I}}^{i}, \bar{w}_{\mathrm{I}}^{i}\right\} \vdash \bar{w}^{i}$ such that $\left|\bar{w}_{\mathrm{I}}^{i}\right|=1$. In (A.4) one should take into account that $\left|\bar{w}_{\text {II }}^{i-1}\right|=r_{i-1}$ while $\left|\bar{w}_{\text {II }}^{i}\right|=\left|\bar{w}^{i}\right|=r_{i}+1$.

The sum over $\ell$ in $(\mathrm{A} .3$ ) can be rewritten as sum over partitions. One can replace the ratio

$$
\frac{f\left(t_{\ell}^{i}, \bar{t}_{\ell}^{i}\right)}{f\left(t_{\ell}^{i}, \bar{t}^{i-1}\right)}=\frac{f\left(t_{\ell}^{i}, \bar{w}_{\mathrm{II}}^{i}\right)}{f\left(t_{\ell}^{i}, \bar{w}^{i-1}\right)}
$$


where $\bar{w}_{\mathrm{II}}^{i}=\left\{z, \bar{t}_{\ell}^{i}\right\}$ and add to the sum over $\ell$ the zero term proportional to

$$
\frac{f\left(z, \bar{t}^{i}\right)}{f\left(z, \bar{w}^{i-1}\right)}=0
$$

The sum over $\ell$ transforms into

$$
\sum_{\ell=1}^{r_{i}} \frac{f\left(t_{\ell}^{i}, \bar{t}_{\ell}^{i}\right)}{f\left(t_{\ell}^{i}, \bar{t}^{i-1}\right)}(\cdot)=\sum_{\text {part }} \frac{f\left(\bar{w}_{\mathrm{I}}^{i}, \bar{w}_{\mathrm{II}}^{i}\right)}{f\left(\bar{w}_{\mathrm{I}}^{i}, \bar{w}_{\mathrm{II}}^{i-1}\right)} \frac{1}{f\left(\bar{w}_{\mathrm{I}}^{i}, \bar{w}_{\mathrm{I}}^{i-1}\right)}(\cdot),
$$

where sum runs over partitions $\left\{\bar{w}_{\mathrm{I}}^{i}, \bar{w}_{\mathrm{II}}^{i}\right\} \vdash \bar{w}^{i}$ such that $\left|\bar{w}_{\mathrm{I}}^{i}\right|=1$. Subtracting A.3 from (A.4) and using (3.10) one gets the action (3.15) with index $i$ replaced by $i+1$.

Let us check that for $j>i+1$ the terms proportional to $\kappa_{i+1}$ in (A.2) vanish. The terms which are proportional to $\kappa_{i+1}$ in the action of $T_{i, j}(z) \mathrm{T}_{i+1, i}^{-}[0] \mathbb{B}(\bar{t})$ is

$$
\begin{aligned}
\left.\mathrm{T}_{i, j}(z) \mathrm{T}_{i+1, i}^{-}[0] \mathbb{B}(\bar{t})\right|_{\kappa_{i}=0} & =-\left(q-q^{-1}\right) \kappa_{i+1} q^{r_{i+1}-r_{i}} \lambda_{1}(z) \times \\
& \times \sum_{\text {part }} \mathbb{B}\left(\bar{w}_{\text {II }}\right) \mathcal{A}_{i, j}\left(\bar{w}_{\mathrm{I}} ; \bar{w}_{\mathrm{II}} ; \bar{w}_{\mathrm{III}}\right) \frac{f\left(\bar{w}_{\mathrm{II}}^{i}, \bar{w}_{\mathrm{II}}^{i}\right)}{f\left(\bar{w}_{\mathrm{II}}^{i+1}, \bar{w}_{\mathrm{II}}^{i}\right)} \frac{q}{f\left(\bar{w}_{\mathrm{II}}^{i+1}, \bar{w}_{\mathrm{II}}^{i}\right)},
\end{aligned}
$$

where we again present the sum over $\ell$ after the action of zero mode operator $\mathrm{T}_{i+1, i}^{-}[0]$ as sum over partitions of the set $\left\{\bar{w}_{\mathrm{II}}^{i}, \bar{w}_{\mathrm{mI}}^{i}\right\} \vdash \bar{w}^{i}=\left\{z, \bar{t}^{i}\right\}$ with $\left|\bar{w}_{\mathrm{mI}}^{i}\right|=1$. The action in inverse order can be written in the same form as (A.5) since $\left|\bar{w}_{\text {II }}^{i}\right|=r_{i}$ and $\left|\bar{w}_{\text {II }}^{i+1}\right|=$ $r_{i+1}+1$ and coefficient at $\kappa_{i+1}$ in the left hand side of (A.2) vanishes.

In the case $j=i+1$ the action $\mathrm{T}_{i, i+1}(z) \mathrm{T}_{i+1, i}^{-}[0] \mathbb{B}(\bar{t})$ is given by equality (A.5), where the set $\bar{w}^{i+1}$ is divided into subsets $\bar{w}_{\text {II }}^{i+1}$ and $\bar{w}_{\text {II }}^{i+1}$ with cardinality $\left|\bar{w}_{\text {II }}^{i+1}\right|=r_{i+1}$. The action in inverse order $\mathrm{T}_{i+1, i}^{-}[0] \mathrm{T}_{i, i+1}(z) \mathbb{B}(\bar{t})$ is

$$
\begin{aligned}
\left.\mathrm{T}_{i+1, i}^{-}[0] \mathrm{T}_{i, i+1}(z) \mathbb{B}(\bar{t})\right|_{\kappa_{i}=0}= & -\left(q-q^{-1}\right) \kappa_{i+1} q^{r_{i+1}-r_{i}} \lambda_{1}(z) \times \\
& \times \sum_{\text {part }} \mathbb{B}\left(\bar{w}_{\text {II }}\right) \mathcal{A}_{i, i+1}\left(\bar{w}_{\mathrm{I}} ; \bar{w}_{\text {II }} ; \bar{w}_{\text {III }}\right) \frac{f\left(\bar{w}_{\text {II }}^{i}, \bar{w}_{\text {III }}^{i}\right)}{f\left(\bar{w}_{\text {II }}^{i+1}, \bar{w}_{\text {III }}^{i}\right)} .
\end{aligned}
$$

Subtracting (A.5) at $j=i+1$ from (A.6) and using (3.11) one gets from (A.2 the action of the diagonal monodromy entry $T_{i, i}(z)$ onto off-shell Bethe vector $\mathbb{B}(\bar{t})$.

\section{B Proof of the proposition 3.4}

In this appendix we prove recurrence relations for the off-shell Bethe vectors $\mathbb{B}(\bar{t})$ formulated in the proposition 3.4. Particular cases of these relations mentioned in the corollary 3.5 were found in [5] in the framework of hierarchical Bethe ansatz. Note that in [5] normalization of the off-shell Bethe vectors was

$$
\prod_{i=1}^{N-1} \beta_{i}\left(\bar{t}^{i}\right)^{-1} \mathbb{B}(\bar{t})
$$


To prove proposition 3.4 one can use the action formulas (3.15) and verify that (3.18) becomes identities. Since the monodromy matrix entries $T_{i, j}(z)$ in (3.18) are upper-triangular $i<j$ we substitute in the right hand side of this equality the action given by (3.17):

$$
\begin{aligned}
\sum_{\text {part }} \sum_{i=1}^{\ell} \sum_{j=\ell+1}^{N} \frac{\lambda_{1}(z)}{\lambda_{\ell}(z)} \frac{\mathbb{B}\left(\left\{\bar{w}_{\mathrm{II}}^{s}\right\}_{1}^{i-1},\left\{z, \bar{t}_{\mathrm{II}}^{s}\right\}_{i}^{\ell-1},\left\{z, \bar{t}^{\ell}\right\} ;\left\{z, \bar{t}_{\mathrm{II}}^{s}\right\}_{\ell+1}^{j-1} ;\left\{\bar{w}_{\mathrm{II}}^{s}\right\}_{j}^{N-1}\right)}{f\left(\bar{t}^{\ell+1}, z\right) f\left(z, \bar{t}^{\ell-1}\right)} \times \\
\quad \times \prod_{p=1}^{i-1} \frac{\beta_{p}\left(\bar{w}_{\mathrm{I}}^{p}\right) f\left(\bar{w}_{\mathrm{I}}^{p}, \bar{w}_{\mathrm{II}}^{p}\right)}{h\left(\bar{w}_{\mathrm{I}}^{p}, \bar{w}_{\mathrm{I}}^{p-1}\right) f\left(\bar{w}_{\mathrm{I}}^{p}, \bar{w}_{\mathrm{II}}^{p-1}\right)} \prod_{p=i}^{\ell-1} \frac{\beta_{p}\left(\bar{t}_{\mathrm{I}}^{p}\right) \tilde{g}\left(\bar{t}_{\mathrm{I}}^{p+1}, \bar{t}_{\mathrm{I}}^{p}\right) f\left(\bar{t}_{\mathrm{I}}^{p}, \bar{t}_{\mathrm{II}}^{p}\right)}{f\left(\bar{t}_{\mathrm{I}}^{p}, \bar{t}^{p-1}\right)} \times \\
\times \prod_{p=\ell+1}^{j-1} \frac{g\left(\bar{t}_{\mathrm{II}}^{p}, \bar{t}_{\mathrm{II}}^{p-1}\right) f\left(\bar{t}_{\mathrm{II}}^{p}, \bar{t}_{\mathrm{II}}^{p}\right)}{f\left(\bar{t}^{p+1}, \bar{t}_{\mathrm{III}}^{p}\right)} \prod_{p=j}^{N-1} \frac{f\left(\bar{w}_{\mathrm{II}}^{p}, \bar{w}_{\mathrm{II}}^{p}\right)}{\tilde{h}\left(\bar{w}_{\mathrm{II}}^{p+1}, \bar{w}_{\mathrm{III}}^{p}\right) f\left(\bar{w}_{\mathrm{II}}^{p+1}, \bar{w}_{\mathrm{III}}^{p}\right)},
\end{aligned}
$$

where sum over partitions of the sets $\left\{\bar{t}^{s}\right\}_{i}^{\ell-1},\left\{\bar{t}^{s}\right\}_{\ell+1}^{j-1}$ are described in propositions 3.4 and of the sets $\left\{\bar{w}^{s}\right\}_{1}^{i-1},\left\{\bar{w}^{s}\right\}_{j}^{N-1}$ in proposition 3.2 respectively. Recall that according to these rules $\bar{w}_{\mathrm{I}}^{0}=\bar{t}_{\mathrm{I}}^{\ell}=\bar{t}_{\mathrm{II}}^{\ell}=\bar{w}_{\mathrm{II}}^{N}=\{z\}$. Our goal is to rewrite the sum over partitions of the sets $\left\{\bar{t}^{s}\right\}_{i}^{\ell-1}$ and $\left\{\bar{t}^{s}\right\}_{\ell+1}^{j-1}$ in $(\overline{B .2})$ as sum over partitions of the sets $\left\{\bar{w}^{s}\right\}_{i}^{\ell-1}=\left\{z, \bar{t}^{s}\right\}_{i}^{\ell-1}$ and $\left\{\bar{w}^{s}\right\}_{\ell+1}^{j-1}=\left\{z, \bar{t}^{s}\right\}_{\ell+1}^{j-1}$.

To do this we transform second and third lines of $(\underline{B .2})$ as follows

$$
\begin{aligned}
& \times \prod_{p=1}^{i-1} \frac{\beta_{p}\left(\bar{w}_{\mathrm{I}}^{p}\right) f\left(\bar{w}_{\mathrm{I}}^{p}, \bar{w}_{\mathrm{II}}^{p}\right)}{h\left(\bar{w}_{\mathrm{I}}^{p}, \bar{w}_{\mathrm{I}}^{p-1}\right) f\left(\bar{w}_{\mathrm{I}}^{p}, \bar{w}_{\mathrm{II}}^{p-1}\right)} \prod_{p=i}^{\ell-1} \frac{\beta_{p}\left(\bar{t}_{\mathrm{I}}^{p}\right) \tilde{g}\left(\bar{t}_{\mathrm{I}}^{p+1}, \bar{t}_{\mathrm{I}}^{p}\right) f\left(\bar{t}_{\mathrm{I}}^{p},\left\{z, \bar{t}_{\mathrm{II}}^{p}\right\}\right)}{f\left(\bar{t}_{\mathrm{I}}^{p},\left\{z, \bar{t}^{p-1}\right\}\right)} \times \\
& \times \prod_{p=\ell+1}^{j-1} \frac{g\left(\bar{t}_{\mathrm{II}}^{p}, \bar{t}_{\mathrm{II}}^{p-1}\right) f\left(\left\{z, \bar{t}_{\mathrm{II}}^{p}\right\}, \bar{t}_{\mathrm{II}}^{p}\right)}{f\left(\left\{z, \bar{t}^{p+1}\right\}, \bar{t}_{\mathrm{II}}^{p}\right)} \prod_{p=j}^{N-1} \frac{\tilde{g}\left(\bar{w}_{\mathrm{II}}^{p+1}, \bar{w}_{\mathrm{II}}^{p}\right) f\left(\bar{w}_{\mathrm{II}}^{p}, \bar{w}_{\mathrm{II}}^{p}\right)}{f\left(\bar{w}^{p+1}, \bar{w}_{\mathrm{III}}^{p}\right)} .
\end{aligned}
$$

Let us at the moment split the values of the boundary sets $\bar{t}_{\mathrm{I}}^{\ell}=\bar{t}_{\mathrm{II}}^{\ell}=\left\{z^{\prime}\right\}$ and $\bar{w}_{\mathrm{I}}^{0}=\bar{w}_{\mathrm{II}}^{N}=\{z\}$ with $z \neq z^{\prime}$. Then the sum over partitions in (B.2) can be written as sum over partitions $\left\{\bar{w}_{\mathrm{I}}^{s}, \bar{w}_{\mathrm{II}}^{s}\right\}_{1}^{\ell-1} \vdash\left\{\bar{w}^{s}\right\}_{1}^{\ell-1}=\left\{z, \bar{t}^{s}\right\}_{1}^{\ell-1}$ and $\left\{\bar{w}_{\mathrm{II}}^{s}, \bar{w}_{\mathrm{III}}^{s}\right\}_{\ell+1}^{N-1} \vdash\left\{\bar{w}^{s}\right\}_{\ell+1}^{N-1}=$ $\left\{z, \bar{t}^{s}\right\}_{\ell+1}^{N-1}$

$$
\begin{aligned}
\sum_{\text {part }} \frac{\mathbb{B}\left(\left\{\bar{w}_{\mathrm{II}}^{s}\right\}_{1}^{\ell-1},\left\{z^{\prime}, \bar{t}^{\ell}\right\},\left\{\bar{w}_{\mathrm{II}}^{s}\right\}_{\ell+1}^{N-1}\right)}{f\left(\bar{t}^{\ell+1}, z^{\prime}\right) f\left(z^{\prime}, \bar{t}^{\ell-1}\right)} \times \\
\quad \times \frac{\lambda_{1}(z)}{\lambda_{\ell}(z)} \sum_{i=1}^{\ell} \prod_{p=1}^{i-1} g\left(\bar{w}_{\mathrm{I}}^{p}, \bar{w}_{\mathrm{I}}^{p-1}\right) \prod_{p=i}^{\ell-1} \tilde{g}\left(\bar{w}_{\mathrm{I}}^{p+1}, \bar{w}_{\mathrm{I}}^{p}\right) \prod_{p=1}^{\ell-1} \frac{\beta_{p}\left(\bar{w}_{\mathrm{I}}^{p}\right) f\left(\bar{w}_{\mathrm{I}}^{p}, \bar{w}_{\mathrm{II}}^{p}\right)}{f\left(\bar{w}_{\mathrm{I}}^{p}, \bar{w}^{p-1}\right)} \times \\
\quad \times \sum_{j=\ell+1}^{N} \prod_{p=\ell+1}^{j-1} g\left(\bar{w}_{\mathrm{II}}^{p}, \bar{w}_{\mathrm{II}}^{p-1}\right) \prod_{p=j}^{N-1} \tilde{g}\left(\bar{w}_{\mathrm{II}}^{p+1}, \bar{w}_{\mathrm{III}}^{p}\right) \prod_{p=\ell+1}^{N-1} \frac{f\left(\bar{w}_{\mathrm{II}}^{p}, \bar{w}_{\mathrm{II}}^{p}\right)}{f\left(\bar{w}^{p+1}, \bar{w}_{\mathrm{II}}^{p}\right)},
\end{aligned}
$$

where the boundary sets $\bar{w}_{\mathrm{I}}^{\ell}=\bar{t}_{\mathrm{I}}^{\ell}=\left\{z^{\prime}\right\}$ and $\bar{w}_{\mathrm{II}}^{\ell}=\bar{t}_{\mathrm{II}}^{\ell}=\left\{z^{\prime}\right\}$ are fixed. The terms in the sum over partitions vanishes when either $\bar{w}_{\mathrm{I}}^{s}=\{z\}$ for $s=i, \ldots, \ell-1$ or $\bar{w}_{\mathrm{III}}^{s}=\{z\}$ for $s=\ell+1, \ldots, j-1$ because either $f\left(z, \bar{w}^{s-1}\right)^{-1}=0$ or $f\left(\bar{w}^{s+1}, z\right)^{-1}=0$.

Using a trivial relation between rational functions

$$
\tilde{g}(x, y)=g(x, y) \frac{y}{x}
$$


one can calculate the sums over $i$ and $j$ in (B.4) to obtain

$$
\begin{aligned}
& \sum_{\text {part }} \frac{\mathbb{B}\left(\left\{\bar{w}_{\mathrm{II}}^{s}\right\}_{1}^{\ell-1},\left\{z^{\prime}, \bar{t}^{\ell}\right\},\left\{\bar{w}_{\mathrm{II}}^{s}\right\}_{\ell+1}^{N-1}\right)}{f\left(\bar{t}^{\ell+1}, z^{\prime}\right) f\left(z^{\prime}, \bar{t}^{\ell-1}\right)} \frac{g\left(z^{\prime}, \bar{w}_{\mathrm{I}}^{\ell-1}\right)}{g\left(z^{\prime}, z\right)} \frac{g\left(\bar{w}_{\mathrm{II}}^{\ell+1}, z^{\prime}\right)}{g\left(z, z^{\prime}\right)} \times \\
& \quad \times \frac{\lambda_{1}(z)}{\lambda_{\ell}(z)} \prod_{p=1}^{\ell-1} \frac{\beta_{p}\left(\bar{w}_{\mathrm{I}}^{p}\right) f\left(\bar{w}_{\mathrm{I}}^{p}, \bar{w}_{\mathrm{II}}^{p}\right)}{f\left(\bar{w}_{\mathrm{I}}^{p}, \bar{w}_{\mathrm{II}}^{p-1}\right) h\left(\bar{w}_{\mathrm{I}}^{p}, \bar{w}_{\mathrm{I}}^{p-1}\right)} \prod_{p=\ell+1}^{N-1} \frac{f\left(\bar{w}_{\mathrm{II}}^{p}, \bar{w}_{\mathrm{II}}^{p}\right)}{f\left(\bar{w}_{\mathrm{II}}^{p+1}, \bar{w}_{\mathrm{II}}^{p}\right) h\left(\bar{w}_{\mathrm{II}}^{p+1}, \bar{w}_{\mathrm{II}}^{p}\right)} .
\end{aligned}
$$

When $z^{\prime} \rightarrow z$ in this sum over partitions only the term survives such that $\bar{w}_{\mathrm{I}}^{s}=\{z\}$ for all $s=1, \ldots, \ell-1$ and $\bar{w}_{\mathrm{II}}^{s}=\{z\}$ for all $s=\ell+2, \ldots, N-1$. Taking into account that according to the proposition $3.2 \bar{w}_{\mathrm{II}}^{0}=\bar{w}_{\mathrm{II}}^{N}=\varnothing$ and the fact that

$$
\prod_{p=1}^{\ell-1} \beta_{p}(z)=\frac{\lambda_{\ell}(z)}{\lambda_{1}(z)}
$$

one concludes that (B.5) is equal to $\mathbb{B}\left(\left\{\bar{t}^{s}\right\}_{1}^{\ell-1},\left\{z, \bar{t}^{\ell}\right\},\left\{\bar{t}^{s}\right\}_{\ell+1}^{N-1}\right)$ so (3.18) becomes identity.

\section{References}

[1] L. D. Faddeev, How Algebraic Bethe Ansatz works for integrable model, in: Les Houches Lectures Quantum Symmetries, eds A. Connes et al, North Holland, (1998) 149, arXiv:hep-th/9605187.

[2] V. G. Drinfeld. Quantum groups, J. Soviet Math., 41:2 (1988) 898-915.

[3] N.Yu. Reshetikhin, M.A. Semenov-Tian-Shansky. Central extension of quantum groups. Lett. Math. Phys. 19 (1990) 133-142.

[4] A. Hutsalyuk, A. Liashyk, S. Z. Pakuliak, E. Ragoucy, N. A. Slavnov. Actions of the monodromy matrix elements onto $\mathfrak{g l}(m \mid n)$-invariant Bethe vectors. J. Stat. Mech. (2020) 093104.

[5] A. Hutsalyuk, A. Liashyk, S. Z. Pakuliak, E. Ragoucy, N. A. Slavnov. Scalar products and norm of Bethe vectors for integrable models based on $U_{q}\left(\widehat{\mathfrak{g l}}_{m}\right)$. SciPost (2018) 4006.

[6] A. Os'kin, S. Pakuliak, A. Silantyev, On the universal weight function for the quantum affine algebra $U_{q}(\mathfrak{g l}(N))$, Algebra and Analysis 21 n.4 (2009) 196-240.

[7] A. Hutsalyuk, A. Liashyk, S. Z. Pakuliak, E. Ragoucy, N. A. Slavnov. Current presentation for the super-Yangian double $D Y(\mathfrak{g l}(m \mid n))$ and Bethe vectors. Russ. Math. Surv. (2017) 72 33, doi:10.1070/RM9754.

[8] A. Liashyk, S. Z. Pakuliak. Algebraic Bethe ansatz for $\mathfrak{o}_{2 n+1}$-invariant integrable models. Theor. and Math. Phys. 206(1) (2021) 19-39. 
[9] J. Ding, I. Frenkel. Isomorphism of two realizations of quantum affine algebra $U_{q}(\mathfrak{g l}(n))$, Comm. Math. Phys. 156 (1993), 277-300, DOI : 10.1007/BF02098484

[10] V. G. Drinfeld. A new realization of Yangians and of quantum affine algebras, Soviet Math. Dokl. 36 (1988) 212-216.

[11] Khoroshkin, S., Tolstoy, V. On Drinfeld realization of quantum affine algebras. Journal of Geometry and Physics 11 (1993), 101-108, DOI: $10.1016 / 0393-0440$ (93) 90070-U

[12] B. Enriquez, S. Khoroshkin, S. Pakuliak, Weight functions and Drinfeld currents, Comm. Math. Phys. 276 (2007) 691-725.

[13] A. Liashyk, S. Z. Pakuliak. On the R-matrix realization of quantum loop algebras. arXiv:2106.10666

[14] S. Khoroshkin, S. Pakuliak, A computation of an universal weight function for the quantum affine algebra $U_{q}(\mathfrak{g l}(N))$, J. of Mathematics of Kyoto University, 48 n.2 (2008) 277-321.

[15] L. Frappat, S. Khoroshkin, S. Pakuliak, E. Ragoucy, Bethe Ansatz for the Universal Weight Function, Ann. H. Poincarre 10 (2009) 513, arXiv:0810.3135. 